\title{
PROPRIÉTÉS (Q) ET (C). VARIÉTÉ COMMUTANTE
}

\author{
Par Jean-Yves Charbonnel
}

\begin{abstract}
RÉSUmÉ. - Soient $X$ une variété algébrique complexe, lisse, irréductible, $E$ et $F$ deux espaces vectoriels complexes de dimension finie et $\mu$ un morphisme de $X$ dans l'espace $\operatorname{Lin}(E, F)$ des applications linéaires de $E$ dans $F$. Pour $x \in X$, on note $E(x)$ et $x \cdot E$ le noyau et l'image de $\mu(x), \bar{\mu}_{x}$ le morphisme de $X$ dans $\operatorname{Lin}(E(x), F /(x \cdot E))$ qui associe à $y$ l'application linéaire $v \mapsto \mu(y)(v)+x \cdot E$. Soit $\mathrm{i}_{\mu}$ la dimension minimale de $E(x)$. On dit que $\mu$ a la propriété $(\mathbf{R})$ en $x$ si $\mathrm{i}_{\bar{\mu}_{x}}$ est inférieur à $\mathrm{i}_{\mu}$. Soient $F^{*}$ le dual de $F, \mathrm{~S}(F)$ l'algèbre symétrique de $F, \mathcal{I}_{\mu}$ l'idéal de $\mathcal{O}_{X} \otimes_{\mathbb{C}} \mathrm{S}(F)$ engendré par les fonctions $\left(x, v^{\prime}\right) \mapsto\left\langle v^{\prime}, \mu(x)(v)\right\rangle$ où $v$ est dans $E$ et $\mathfrak{C}_{\mu}$ la sous-variété des zéros dans $X \times F^{*}$ de $\mathcal{I}_{\mu}$. Désignant par $\sqrt{\mathcal{I}_{\mu}}$ le radical de $\mathcal{I}_{\mu}$, par $\Sigma$ le support de $\sqrt{\mathcal{I}_{\mu}} / \mathcal{I}_{\mu}$ dans $X \times F^{*}$ et par $S$ la projection de $\Sigma$ sur $X$, le premier résultat principal de ce mémoire dit que sous deux conditions techniques sur $\mu, S$ est une partie fermée de $X$ dont la codimension est supérieure à 2 si et seulement si l'adhérence de l'ensemble des points de $X$ en lesquels $\mu$ n'a pas la propriété $(\mathbf{R})$, a une codimension supérieure à 2 .

Soit $\mathfrak{g}$ une algèbre de Lie. On dit que $\mathfrak{g}$ a la propriété $(\mathbf{C})$ en l'élément $\xi$ de $\mathfrak{g}$ si l'application adjointe de $\mathfrak{g}$ dans l'espace des endomorphismes linéaires de $\mathfrak{g}$ a la propriété $(\mathbf{R})$ en $\xi$ et que $\mathfrak{g}$ a la propriété $(\mathbf{Q})$ en l'élément $v^{\prime}$ de $\mathfrak{g}^{*}$ si l'application coadjointe de $\mathfrak{g}^{*}$ dans $\operatorname{Lin}\left(\mathfrak{g}, \mathfrak{g}^{*}\right)$ a la propriété $(\mathbf{R})$ en $v^{\prime}$. L'algèbre $\mathfrak{g}$ a la propriété $(\mathbf{Q})$ en $v^{\prime}$ si et seulement si l'indice du stabilisateur $\mathfrak{g}\left(v^{\prime}\right)$ de $v^{\prime}$ est égal à l'indice de $\mathfrak{g}$. Le deuxième résultat principal dit qu'une algèbre de Lie réductive a la propriété $(\mathbf{Q})$ en tout point de $\mathfrak{g}^{*}$.
\end{abstract}

Texte reçu le 8 novembre 2001, révisé les 26 juillet 2002 et 30 janvier 2003, accepté le 30 juin 2003

Jean-Yves Charbonnel, Université Paris 7 - CNRS, Institut de Mathématiques de Jussieu, Théorie des groupes, Case 7012, 2 Place Jussieu, 75251 Paris Cedex 05 (France)

E-mail : jyc@math.jussieu.fr

Classification mathématique par sujets (2000). — 14A10, 14L17, 22E20, 22E46.

Mots clefs. - Indice, algèbre de Lie, endomorphisme linéaire, codimension, variété algébrique. 


\begin{abstract}
Properties $(\mathbf{Q})$ and $(\mathbf{C})$. Commuting variety). - Let $X$ be a complex, smooth, irreducible algebraic variety, $E$ and $F$ be two finite dimensional complex vector spaces and $\mu$ be a morphism from $X$ to the space $\operatorname{Lin}(E, F)$ of linear maps from $E$ to $F$. For $x$ in $X$, we denote by $E(x)$ and $x \cdot E$ the kernel and the image of $\mu(x)$, and by $\bar{\mu}_{x}$ the morphism from $X$ to $\operatorname{Lin}(E(x), F /(x \cdot E))$ which associates to $y$ the linear map $v \mapsto \mu(y)(v)+x \cdot E$. Let $\mathrm{i}_{\mu}$ be the smallest dimension of $E(x)$. We say that $\mu$ has property $(\mathbf{R})$ at $x$ if $\mathrm{i}_{\bar{\mu}}$ is not greater than $\mathrm{i}_{\mu}$. Let $F^{*}$ be the dual of $F$, $\mathrm{S}(F)$ be the symmetric algebra of $F, \mathcal{I}_{\mu}$ be the ideal of $\mathcal{O}_{X} \otimes_{\mathbb{C}} \mathrm{S}(F)$ generated by the functions $\left(x, v^{\prime}\right) \mapsto\left\langle v^{\prime}, \mu(x)(v)\right\rangle$ where $v$ is in $E$ and $\mathfrak{C}_{\mu}$ be the subvariety of zeros in $X \times F^{*}$ of $\mathcal{I}_{\mu}, \sqrt{\mathcal{I}_{\mu}}$ be the radical of $\mathcal{I}_{\mu}, \Sigma$ be the support of $\sqrt{\mathcal{I}_{\mu}} / \mathcal{I}_{\mu}$ in $X \times F^{*}$ and $S$ be the projection of $\Sigma$ on $X$. The first main result says that under two technical conditions on $\mu, S$ is a closed subset of $X$ whose codimension is at least equal to 2 if and only if the closure of the subset of points in $X$ at which $\mu$ has not property $(\mathbf{R})$, has codimension at least equal to 2 .

Let $\mathfrak{g}$ be a Lie algebra. We say that $\mathfrak{g}$ has the property $(\mathbf{C})$ at the element $\xi$ of $\mathfrak{g}$ if the adjoint map from $\mathfrak{g}$ to the space of linear endomorphisms of $\mathfrak{g}$ has the property $(\mathbf{R})$ at $\xi$ and that $\mathfrak{g}$ has the property $(\mathbf{Q})$ at the element $v^{\prime}$ of $\mathfrak{g}^{*}$ if the coadjoint map from $\mathfrak{g}^{*}$ to $\operatorname{Lin}\left(\mathfrak{g}, \mathfrak{g}^{*}\right)$ has the property $(\mathbf{R})$ at $v^{\prime}$. The algebra $\mathfrak{g}$ has the property $(\mathbf{Q})$ at $v^{\prime}$ if and only if the index of the stabilizer $\mathfrak{g}\left(v^{\prime}\right)$ of $v^{\prime}$ is equal to the index of $\mathfrak{g}$. The second main result says that any reductive Lie algebra has property $(\mathbf{Q})$ at any point of $\mathfrak{g}^{*}$.
\end{abstract}

\title{
1. Introduction
}

Le corps de base est le corps $\mathbb{C}$ des nombres complexes. On fixe une fois pour toutes une variété algébrique $X$, lisse et irréductible, $E$ et $F$ deux espaces vectoriels de dimension finie et $\mu$ un morphisme de $X$ dans l'espace $\operatorname{Lin}(E, F)$ des applications linéaires de $E$ dans $F$. Pour tout point $x$ de $X$, on note respectivement $E(x)$ et $x \cdot E$ le noyau et l'image de $\mu(x)$. On utilise la topologie de Zariski sur les variétés algébriques considérées. On appelle grand ouvert de $X$ un ouvert dont le complémentaire est de codimension supérieure à 2 dans $X$. Sauf mention contraire, on entend par point de $X$ un point fermé. Conformément à l'usage, $\mathbb{C}[X]$ désigne l'anneau des fonctions régulières sur $X, \mathcal{O}_{X}$ désigne le faisceau structural de $X$ et $\mathcal{O}_{X, x}$ désigne l'anneau local au point $x$ de $X$.

Soient $\theta$ le morphisme de $\mathcal{O}_{X}$-modules

$$
\mathcal{O}_{X} \otimes_{\mathbb{C}} E \longrightarrow \mathcal{O}_{X} \otimes_{\mathbb{C}} F, \quad \varphi \otimes v \longmapsto(x \mapsto \varphi(x) \mu(x)(v))
$$

et $\mathcal{M}_{\mu}$ le sous- $\mathcal{O}_{X}$-module des sections locales $\varphi$ de $\mathcal{O}_{X} \otimes_{\mathbb{C}} F$ qui satisfont la condition suivante : $x \cdot E$ contient $\varphi(x)$ pour tout $x$ dans le domaine de définition de $\varphi$. Par définition, l'image de $\theta$ est un sous- $\mathcal{O}_{X}$-module de $\mathcal{M}_{\mu}$. On s'intéresse alors à la propriété $(\mathbf{P})$ pour le morphisme $\mu$ : l'image de $\theta$ et le faisceau $\mathcal{M}_{\mu}$ ont même restriction à un grand ouvert de $X$. L'étude de la propriété $(\mathbf{P})$ dégage la notion d'ouverts admissibles pour $\mu$ qui est donnée dans la définition 2.7. Le théorème 2.9 dit alors que $\mu$ a la propriété $(\mathbf{P})$ si la réunion des ouverts admissibles pour $\mu$ est un grand ouvert de $X$. Considérant

TOME $132-2004-\mathrm{N}^{\mathrm{O}} 4$ 
la dimension minimale $\mathrm{i}_{\mu}$ des noyaux $E(x)$, on introduit dans la définition 3.1 la propriété $(\mathbf{R})$ en un point $x$ de $X$ pour le morphisme $\mu$. Le théorème 3.3 dit alors que sous deux conditions techniques sur le morphisme $\mu$, la propriété $(\mathbf{P})$ pour le morphisme $\mu$ est équivalente à la propriété $(\mathbf{R})$ pour $\mu$ en tout point d'un grand ouvert de $X$. Soient $F^{*}$ le dual de $F$ et $\mathrm{S}(F)$ l'algèbre symétrique de $F$. On désigne par $\mathfrak{C}_{\mu}$ l'ensemble des points $\left(x, v^{\prime}\right)$ de $X \times F^{*}$ tels que le noyau de $v^{\prime}$ contient $x \cdot E$, et par $\mathcal{I}_{\mu}$ l'idéal de $\mathcal{O}_{X} \otimes_{\mathbb{C}} \mathrm{S}(F)$ engendré par les fonctions $\left(x, v^{\prime}\right) \mapsto\left\langle v^{\prime}, \mu(x)(v)\right\rangle$ où $v$ est dans $V$. Par définition, $\mathcal{I}_{\mu}$ est contenu dans l'idéal de définition de $\mathfrak{C}_{\mu}$ dans $\mathcal{O}_{X} \otimes_{\mathbb{C}} \mathrm{S}(F)$ et $\mathfrak{C}_{\mu}$ est la variété des zéros de $\mathcal{I}_{\mu}$ dans $X \times F^{*}$. La question de savoir si l'idéal $\mathcal{I}_{\mu}$ est un idéal radiciel est bien naturelle; le théorème 4.1 donne une réponse partielle. Désignant par $\sqrt{\mathcal{I}_{\mu}}$ le radical de $\mathcal{I}_{\mu}$, le théorème 4.1 dit que sous les conditions techniques du théorème 3.3 , les faisceaux $\mathcal{I}_{\mu}$ et $\sqrt{\mathcal{I}_{\mu}}$ sur $X$ ont même restriction à un grand ouvert de $X$ si et seulement si $\mu$ a la propriété $(\mathbf{R})$ en tout point d'un grand ouvert de $X$.

On applique enfin ces résultats dans le cas particulier où $X$ est la variété sous-jacente à une algèbre de Lie $\mathfrak{g}$ ou à son dual $\mathfrak{g}^{*}$. Dans le cas où $X$ est égal à $\mathfrak{g}$, on note $\mu$ le morphisme qui à $x$ associe l'endomorphisme ad $x$ de $\mathfrak{g}$ et dans le cas où $X$ est égal à $\mathfrak{g}^{*}$, on note $\nu$ l'application qui à l'élément $x$ de $\mathfrak{g}^{*}$ associe l'application linéaire $v \mapsto v \cdot x$ de $\mathfrak{g}$ dans $\mathfrak{g}^{*}$ où $v \cdot x$ désigne l'action coadjointe de $v$ sur $x$. La propriété $(\mathbf{R})$ pour $\mu$ est appelée propriété $(\mathbf{C})$ pour l'algèbre de Lie $\mathfrak{g}$ et la propriété $(\mathbf{R})$ pour $\nu$ est appelée propriété $(\mathbf{Q})$ pour l'algèbre de Lie $\mathfrak{g}$. La variété $\mathfrak{C}_{\mu}$ est la variété $\mathfrak{C}_{\mathfrak{g}}$ des éléments $\left(\xi, v^{\prime}\right)$ de $\mathfrak{g} \times \mathfrak{g}^{*}$ où $\xi$ appartient au stabilisateur de $v^{\prime}$ dans $\mathfrak{g}$ et la variété $\mathfrak{C}_{\nu}$ est isomorphe à la variété $\mathfrak{C}_{\mathfrak{g}}$. On désigne par $I_{\mathfrak{g}}$ l'espace des sections globales de $\mathcal{I}_{\mu}$. On a alors le théorème :

THÉORÈmE. - Soit $\Sigma$ le support dans $\mathfrak{g} \times \mathfrak{g}^{*} d u \mathrm{~S}\left(\mathfrak{g}^{*}\right) \otimes_{\mathbb{C}} \mathrm{S}(\mathfrak{g})$-module $\sqrt{I_{\mathfrak{g}}} / I_{\mathfrak{g}}$.

(i) L'algèbre de Lie $\mathfrak{g}$ a la propriété $(\mathbf{C})$ en tout point d'un grand ouvert de $\mathfrak{g}$ si et seulement si la projection de $\Sigma$ sur $\mathfrak{g}$ est une sous-variété fermée de codimension supérieure à 2 de $\mathfrak{g}$.

(ii) L'algèbre de Lie $\mathfrak{g}$ a la propriété $(\mathbf{Q})$ en tout point d'un grand ouvert de $\mathfrak{g}^{*}$ si et seulement si la projection de $\Sigma$ sur $\mathfrak{g}^{*}$ est une sous-variété fermée de codimension supérieure à 2 de $\mathfrak{g}^{*}$.

Par définition, l'algèbre de Lie $\mathfrak{g}$ est dite quadratique lorsque ses modules adjoint et coadjoint sont isomorphes. Les propriétés $(\mathbf{C})$ et $(\mathbf{Q})$ pour une algèbre de Lie quadratique sont donc équivalentes. En outre, dans le cas où $\mathfrak{g}$ est une algèbre de Lie quadratique, la variété $\mathfrak{C}_{\mathfrak{g}}$ s'identifie à la variété des éléments $(\xi, \eta)$ de $\mathfrak{g} \times \mathfrak{g}$ qui commutent entre eux. Pour $\mathfrak{g}$ algèbre de Lie quelconque, on appelera variété commutante de $\mathfrak{g}$ la variété $\mathfrak{C}_{\mathfrak{g}}$ ci-dessus. En général, l'idéal $I_{\mathfrak{g}}$ n'est pas radiciel et la variété $\mathfrak{C}_{\mathfrak{g}}$ n'est pas irréductible. R.W. Richardson a montré dans [4] que $\mathfrak{C}_{\mathfrak{g}}$ est une variété irréductible dans le cas où $\mathfrak{g}$ est une algèbre 
de Lie réductive. On rappelle que l'indice de $\mathfrak{g}$ est la dimension minimale des stabilisateurs dans $\mathfrak{g}$ des éléments de $\mathfrak{g}^{*}$. Dire que $\mathfrak{g}$ a la propriété $(\mathbf{Q})$ en $v^{\prime}$ revient à dire que l'indice de $\mathfrak{g}\left(v^{\prime}\right)$ est égal à l'indice de $\mathfrak{g}$. En général, une algèbre de Lie complexe n'a pas la propriété $(\mathbf{Q})$. L'indice d'une algèbre de Lie réductive est égal à son rang. On montre alors le théorème :

ThÉORÈme. - Soit $\mathfrak{g}$ une algèbre de Lie réductive complexe. Alors l'indice du centralisateur de tout élément de $\mathfrak{g}$ est égal au rang de $\mathfrak{g}$.

Ce résultat avait été conjecturé par A.G. Elashvili. Cette question a été étudiée par D.I. Panyushev dans [3] où il y donne des résultats partiels. En outre, $\mathrm{P}$. Tauvel a montré dans [7] que pour $\mathfrak{g}$ algèbre de Lie réductive, le centralisateur d'un élément de $\mathfrak{g}$ est commutatif si et seulement si cet élément est régulier. La propriété $(\mathbf{Q})$ pour $\mathfrak{g}$ redonne ce résultat car l'indice d'une algèbre commutative est égal à sa dimension. Le point clé de la démonstration de ce théorème est le résultat de J. Dixmier sur les champs de vecteurs adjoints d'une algèbre de Lie réductive [2]. Le cas des algèbres de Lie est la motivation principale de ce mémoire dont la suite se divise en quatre sections :

2) la propriété $(\mathbf{P})$

3) les propriétés $(\mathbf{P})$ et $(\mathbf{R})$,

4) application de la propriété $(\mathbf{R})$,

5) application aux algèbres de Lie.

Je remercie vivement $\mathrm{N}$. Ressayre pour l'intérêt qu'il a porté à ce travail et pour ses nombreuses suggestions. Je lui dois en particulier la présentation et les formulations des définitions des propriétés $(\mathbf{P})$ et $(\mathbf{R})$ et du théorème 2.9 .

\section{La propriété $(P)$}

Soit $\mu$ une application régulière de $X$ dans l'espace $\operatorname{Lin}(E, F)$ des applications linéaires de $E$ dans $F$.

DÉFInition 2.1. - On appelle indice de $\mu$ et on note $\mathrm{i}_{\mu}$ la plus petite des dimensions des noyaux des applications linéaires $\mu(x)$ où $x$ est dans $X$. On désigne par $X_{\mathrm{r}}$ l'ensemble des points $x$ de $X$ pour lesquels $\mathrm{i}_{\mu}$ est la dimension du noyau de $\mu(x)$.

D'après un lemme d'algèbre linéaire :

Soit $L_{r}$ l'ensemble des applications linéaires de rang $r$ de $E$ dans $F$. Alors $L_{r}$ est une partie localement fermée de $\operatorname{Lin}(E, F)$ et son adhérence est la réunion des $L_{s}$ où $s$ est inférieur à $r$. En outre, l'application $u \mapsto$ Ker $u$ est une application régulière de $L_{r}$ dans la grassmannienne $\operatorname{Gr}_{\operatorname{dim} E-r}(E)$ et l'application qui à $u$ associe l'image de $u$ est une application régulière de $L_{r}$ dans $\operatorname{Gr}_{r}(F)$,

TOME $132-2004-\mathrm{N}^{\mathrm{O}} 4$ 
L'ensemble $X_{\mathrm{r}}$ est ouvert dans $X$, l'application $x \mapsto E(x)$ de $X_{\mathrm{r}}$ dans $\operatorname{Gr}_{\mathrm{i}_{\mu}}(E)$ est régulière et l'application $x \mapsto x \cdot E$ de $X_{\mathrm{r}}$ dans $\operatorname{Gr}_{\operatorname{dim} E-\mathrm{i}_{\mu}}(F)$ est régulière. Puisque $X$ est une variété lisse, l'application régulière $x \mapsto E(x)$ de $X_{\mathrm{r}}$ dans $\operatorname{Gr}_{\mathrm{i}_{\mu}}(E)$ se prolonge à un grand ouvert de $X$ d'après [5, ch. VI, th. 1$]$. On note $X_{*}$ la réunion des ouverts de $X$ auxquels l'application $x \mapsto E(x)$ de $X_{\mathrm{r}}$ dans $\operatorname{Gr}_{i_{\mu}}(E)$ a un prolongement régulier. En particulier, $X_{*}$ est un grand ouvert de $X$ qui contient $X_{\mathrm{r}}$ et il existe une application régulière $\alpha_{\mu}$ de $X_{*}$ dans $\operatorname{Gr}_{\mathrm{i}_{\mu}}(E)$ pour laquelle $\alpha_{\mu}(x)$ est égal à $E(x)$ pour tout $x$ dans $X_{\mathrm{r}}$.

DÉFInItion 2.2. - Soit $\theta$ le morphisme de $\mathcal{O}_{X}$-modules :

$$
\mathcal{O}_{X} \otimes_{\mathbb{C}} E \longrightarrow \mathcal{O}_{X} \otimes_{\mathbb{C}} F, \quad \varphi \otimes v \longmapsto(x \mapsto \varphi(x) \mu(x)(v)),
$$

où $\varphi$ est une section locale de $\mathcal{O}_{X}$ et où $v$ est dans $E$. Soit $\mathcal{M}_{\mu}$ le sous- $\mathcal{O}_{X^{-}}$ module de $\mathcal{O}_{X} \otimes_{\mathbb{C}} F$ défini par la condition suivante : pour tout ouvert $V$ de $X$, $\varphi$ est section de $\mathcal{M}_{\mu}$ au-dessus de $V$ si et seulement si $x \cdot E$ contient $\varphi(x)$ pour tout $x$ dans $V$. On dira que $\mu$ a la propriété $(\mathbf{P})$ si $\mathcal{M}_{\mu}$ et l'image de $\theta$ ont même restriction à $X_{*}$.

Dans la suite de cette section, on suppose $X$ égal à $X_{*}$.

2.1. Il est clair que $\mathcal{M}_{\mu}$ contient l'image de $\theta$ et on montre facilement que $\mathcal{M}_{\mu}$ et l'image de $\theta$ ont même restriction à $X_{\mathrm{r}}$.

LEMME 2.3. - (i) La réunion de $X_{\mathrm{r}}$ et des ouverts de $X$ qui satisfont les conditions suivantes

1) $Y$ est un ouvert affine de $X$ tel que $Y \backslash X_{\mathrm{r}}$ est un diviseur premier de $Y$ dont l'idéal de définition dans $\mathbb{C}[Y]$ est engendré par un élément $q$,

2) il existe un sous-espace $\mathfrak{m}$ de $E$ qui est un supplémentaire de $\alpha_{\mu}(x)$ dans $E$ pour tout $x$ dans $Y$,

3) la dimension de $E(x)$ est constante sur $Y \backslash X_{\mathrm{r}}$,

est un grand ouvert de $X$.

(ii) Si $\mathcal{M}_{\mu}$ et l'image de $\theta$ ont même restriction à un grand ouvert de $X$, alors $\mu$ a la propriété $(\mathbf{P})$.

Démonstration. - (i) On note $S$ la réunion du lieu singulier de $X \backslash X_{\mathrm{r}}$ et des composantes irréductibles de $X \backslash X_{\mathrm{r}}$ de codimension supérieure à 2 dans $X$. Alors $X \backslash S$ est un grand ouvert de $X$ et l'intersection de $X \backslash X_{\mathrm{r}}$ avec $X \backslash S$ est une hypersurface lisse; or $X$ est lisse; donc $X \backslash S$ est recouvert par des ouverts qui satisfont la condition 1$)$. Soient $T_{1}, \ldots, T_{\ell}$ les composantes irréductibles de l'intersection de $X \backslash X_{\mathrm{r}}$ et de $X \backslash S$. Pour $i=1, \ldots, \ell$, on désigne par $T_{i}^{\prime}$ l'ensemble des points $x$ de $T_{i}$ pour lesquels $E(x)$ est de dimension minimale. Alors $T_{i} \backslash T_{i}^{\prime}$ est fermé dans $X \backslash S$ et de codimension supérieure à 2 ; donc le complémentaire dans $X \backslash S$ de la réunion des ensembles $T_{1} \backslash T_{1}^{\prime}, \ldots, T_{\ell} \backslash T_{\ell}^{\prime}$ est un grand ouvert de $X$. En outre, il est réunion d'ouverts affines qui satisfont les 
conditions 1) et 3). L'assertion résulte alors de ce que tout ouvert de $X$ est réunion d'ouverts affines qui satisfont la condition 2).

(ii) On suppose que $\mathcal{M}_{\mu}$ et l'image de $\theta$ ont même restriction à un grand ouvert de $X$. Soit $Y$ un ouvert affine de $X$ qui satisfait la condition 2). On note $Y_{\mathrm{r}}$ l'intersection de $Y$ et de $X_{\mathrm{r}}$. Soit $\varphi$ une section de $\mathcal{M}_{\mu}$ au-dessus de $Y$. Alors il existe une application régulière $\psi$ et une seule de $Y_{\mathrm{r}}$ dans $\mathfrak{m}$ qui satisfait l'égalité $\varphi(x)=\mu(x)(\psi(x))$ pour tout $x$ dans $Y_{\mathrm{r}}$. Vu l'arbitraire de $Y$ et de $\varphi$, il s'agit de montrer que $\psi$ a un prolongement régulier à $Y$. Par hypothèse, pour tout ouvert affine $Y_{1}$ d'un grand ouvert $Y^{\prime}$ de $Y$, il existe une application régulière $\psi_{1}$ de $Y_{1}$ dans $E$ qui satisfait l'égalité $\varphi(x)=\mu(x)\left(\psi_{1}(x)\right)$ pour tout $x$ dans $Y_{1}$. Puisque $\alpha_{\mu}(x)$ est un supplémentaire de $\mathfrak{m}$ dans $E$ pour tout $x$ dans $Y_{1}$, il existe une unique application régulière $\psi_{1}^{\prime}$ de $Y_{1}$ de $\mathfrak{m}$ qui satisfait les deux relations

$$
\psi_{1}^{\prime}(x) \in \mathfrak{m} \quad \text { et } \quad \psi_{1}(x)-\psi_{1}^{\prime}(x) \in \alpha_{\mu}(x),
$$

pour tout $x$ dans $Y_{1}$. Pour tout $x$ dans l'intersection de $Y_{1}$ et de $Y_{\mathrm{r}}, \varphi(x)$ est égal à $\mu(x)\left(\psi_{1}^{\prime}(x)\right)$ car $\alpha_{\mu}(x)$ est égal à $E(x)$; par continuité, $\varphi(x)$ est donc égal à $\mu(x)\left(\psi_{1}^{\prime}(x)\right)$ pour tout $x$ dans $Y_{1}$ car $Y_{1}$ est irréductible. Il résulte alors de l'unicité de $\psi$, que $\psi_{1}$ est un prolongement régulier à $Y_{1}$ de la restriction de $\psi$ à l'intersection de $Y_{\mathrm{r}}$ et de $Y_{1}$. Par suite, $\psi$ a un prolongement régulier à $Y^{\prime}$. La variété $Y$ est normale car elle est lisse; donc $\psi$ a un prolongement régulier à $Y$ car $Y^{\prime}$ est un grand ouvert de $Y$.

Il résulte du lemme que $\mu$ a la propriété $(\mathbf{P})$ si et seulement si la restriction de $\mu$ à tout ouvert de $X$ qui satisfait les conditions 1), 2), 3) a la propriété (P).

2.2. Soit $Y$ un ouvert de $X$ qui satisfait les conditions 1), 2), 3) du lemme 2.3. On note $s$ l'entier $\operatorname{dim} E(x)-\mathrm{i}_{\mu}$ où $x$ est dans $Y \backslash X_{\mathrm{r}}$.

DÉFInITION 2.4. - On dira que $Y$ est un ouvert adéquat pour $\mu$ s'il existe des applications régulières $\varepsilon_{1}, \ldots, \varepsilon_{m}$ de $Y$ dans $E$ et des applications régulières $\nu_{1}, \ldots, \nu_{s}$ de $Y$ dans $F$ qui satisfont les conditions :

1) pour tout $x$ dans $Y$ et pour $i=m-s+1, \ldots, m, \mu(x)\left(\varepsilon_{i}(x)\right)$ est égal à $q(x) \nu_{i+s-m}(x)$

2) pour tout $x$ dans $Y$, les éléments $\mu(x)\left(\varepsilon_{1}(x)\right), \ldots, \mu(x)\left(\varepsilon_{m-s}(x)\right)$, $\nu_{1}(x), \ldots, \nu_{s}(x)$ sont linéairement indépendants,

$3)$ pour tout $x$ dans $Y$, les éléments $\mu(x)\left(\varepsilon_{1}(x)\right), \ldots, \mu(x)\left(\varepsilon_{m}(x)\right)$ engendrent le sous-espace $x \cdot E$ de $F$.

LEMME 2.5. - (i) L'ouvert $Y$ est adéquat si et seulement s'il existe des applications régulières $\varepsilon_{1}, \ldots, \varepsilon_{m}$ de $Y$ dans $E$ et des applications régulières $\nu_{1}, \ldots, \nu_{s}$ de $Y$ dans $F$ qui satisfont les conditions 1), 2), 3) de la définition 2.4 et la condition suivante : pour tout $x$ dans $Y, \varepsilon_{1}(x), \ldots, \varepsilon_{m}(x)$ est une base de $\mathfrak{m}$.

TOME $132-2004-\mathrm{N}^{\mathrm{O}} 4$ 
(ii) L'ouvert $Y$ contient un ouvert qui rencontre $Y \backslash X_{\mathrm{r}}$ et qui est adéquat si et seulement si pour toute base $e_{1}, \ldots, e_{m}$ de $\mathfrak{m}$, l'application rationnelle de $Y$ dans $\wedge^{m}(F)$

$$
x \longmapsto q(x)^{-s} \mu(x)\left(e_{1}\right) \wedge \cdots \wedge \mu(x)\left(e_{m}\right),
$$

est une application régulière qui n'est pas identiquement nulle sur $Y \backslash X_{\mathrm{r}}$.

Démonstration. - (i) Il s'agit de montrer que la condition est nécessaire. On suppose $Y$ adéquat pour $\mu$. Alors il existe des applications régulières $\eta_{1}, \ldots, \eta_{m}$ de $Y$ dans $E$ et des applications régulières $\nu_{1}, \ldots, \nu_{s}$ de $Y$ dans $F$ qui satisfont les conditions 1), 2), 3) de la définition 2.4. Pour $i=1, \ldots, m$ et pour $x$ dans $Y$, on désigne par $\varepsilon_{i}(x)$ la projection de $\eta_{i}(x)$ sur $\mathfrak{m}$ parallèlement à $\alpha_{\mu}(x)$. Puisque $\eta_{i}$ est une application régulière, l'application $\varepsilon_{i}$ ainsi définie est régulière. Pour tout $x$ dans $Y, \mu(x)\left(\varepsilon_{i}(x)\right)$ est égal à $\mu(x)\left(\eta_{i}(x)\right)$; donc les applications $\varepsilon_{1}, \ldots, \varepsilon_{m}$ et les applications $\nu_{1}, \ldots, \nu_{s}$ satisfont les conditions 1$), 2$ ), 3 ) de la définition 2.4. En particulier, pour tout $x$ dans l'intersection de $Y$ et de $X_{\mathrm{r}}$, la dimension de $x \cdot E$ est égale à $m$; or $\mathfrak{m}$ et $x \cdot E$ ont même dimension car $\mathfrak{m}$ est un supplémentaire de $E(x)$ dans $E$ pour tout $x$ dans l'intersection de $Y$ et de $X_{\mathrm{r}}$; donc $m$ est la dimension de $\mathfrak{m}$ et la famille $\varepsilon_{1}(x), \ldots, \varepsilon_{m}(x)$ est une base de $\mathfrak{m}$ pour tout $x$ dans l'intersection de $Y$ et de $X_{\mathrm{r}}$.

On suppose qu'il existe un point $x$ de $Y \backslash X_{\mathrm{r}}$ pour lequel $\varepsilon_{1}(x), \ldots, \varepsilon_{m}(x)$ n'est pas une famille libre. Il s'agit d'aboutir à une contradiction. Puisque $m$ est la dimension de $\mathfrak{m}$, l'ensemble des points $x$ de $Y$ qui satisfont l'égalité

$$
\varepsilon_{1}(x) \wedge \cdots \wedge \varepsilon_{m}(x)=0
$$

est une hypersurface de $Y$; or d'après ce qui précède, cette hypersurface est contenue dans $Y \backslash X_{\mathrm{r}}$; donc elle est égale à $Y \backslash X_{\mathrm{r}}$ car $Y \backslash X_{\mathrm{r}}$ est irréductible. Soit $x$ un point de $Y \backslash X_{\mathrm{r}}$ pour lequel la famille $\varepsilon_{1}(x), \ldots, \varepsilon_{m}(x)$ est de rang maximum. Soit $m^{\prime}$ ce rang. Par hypothèse, $m^{\prime}$ est strictement inférieur à $m$. D'après les conditions 1 ) et 2) de la définition $2.4, \varepsilon_{1}(x), \ldots, \varepsilon_{m-s}(x)$ sont linéairement indépendants; donc $m^{\prime}$ est supérieur à $m-s$. Quitte à réordonner les applications $\varepsilon_{1}, \ldots, \varepsilon_{m}$, on peut supposer que la famille $\varepsilon_{1}(x), \ldots, \varepsilon_{m^{\prime}}(x)$ est libre. On peut alors trouver un ouvert affine $Y^{\prime}$ de $Y$, contenant $x$, et des fonctions régulières $a_{1}, \ldots, a_{m^{\prime}}$ sur $Y^{\prime}$ pour lesquelles l'application

$$
y \longmapsto \varepsilon_{m^{\prime}+1}(y)-a_{1}(y) \varepsilon_{1}(y)-\cdots-a_{m^{\prime}}(y) \varepsilon_{m^{\prime}}(y),
$$

est nulle sur $Y^{\prime} \backslash X_{\mathrm{r}}$. Puisque la restriction de $q$ à $Y^{\prime}$ engendre l'idéal de définition de $Y \backslash X_{\mathrm{r}}$ dans $\mathbb{C}\left[Y^{\prime}\right]$, il existe une application régulière $\rho$ de $Y^{\prime}$ dans $\mathfrak{m}$ qui n'est pas identiquement nulle sur $Y^{\prime} \backslash X_{\mathrm{r}}$ et un entier strictement positif $\ell$ qui satisfont l'égalité

$$
\varepsilon_{m^{\prime}+1}=q^{\ell} \rho+\sum_{j=1}^{m^{\prime}} a_{j} \varepsilon_{j} .
$$

BULLETIN DE LA SOCIÉtÉ MATHÉMATIQUE DE FRANCE 
Par suite, il vient

$$
\mu(y)\left(\varepsilon_{m^{\prime}+1}(y)\right)=q(y)^{\ell} \mu(y)(\rho(y))+\sum_{j=1}^{m^{\prime}} a_{j}(y) \mu(y)\left(\varepsilon_{j}(y)\right),
$$

pour tout $y$ dans $Y^{\prime}$. D'après la condition 1 ) de la définition 2.4, on a

$$
\begin{gathered}
q(y) \nu_{m^{\prime}-m+s+1}(y)=q(y)^{\ell} \mu(y)(\rho(y))+\sum_{j=1}^{m-s} a_{j}(y) \mu(y)\left(\varepsilon_{j}(y)\right) \\
+q(y) \sum_{j=m-s+1}^{m^{\prime}} a_{j}(y) \nu_{j-m+s}(y),
\end{gathered}
$$

pour tout $y$ dans $Y^{\prime}$; or d'après la condition 2) de la définition 2.4, pour tout $y$ dans $Y^{\prime} \backslash X_{\mathrm{r}}, \mu(y)\left(\varepsilon_{1}(y)\right), \ldots, \mu(y)\left(\varepsilon_{m-s}(y)\right)$ sont linéairement indépendants; donc $q$ divise $a_{1}, \ldots, a_{m-s}$ dans $\mathbb{C}\left[Y^{\prime}\right]$. En désignant par $b_{1}, \ldots, b_{m-s}$ les quotients respectifs de $a_{1}, \ldots, a_{m-s}$ par $q$, il vient

$$
\begin{aligned}
\nu_{m^{\prime}-m+s+1}(y) & -\sum_{j=m-s+1}^{m^{\prime}} a_{j}(y) \nu_{j-m+s}(y) \\
= & q(y)^{\ell-1} \mu(y)(\rho(y)) \sum_{j=1}^{m-s} b_{j}(y) \mu(y)\left(\varepsilon_{j}(y)\right),
\end{aligned}
$$

pour tout $y$ dans $Y^{\prime}$. Ceci est absurde car d'après les conditions 1), 2), 3) de la définition 2.4, $\nu_{1}(y), \ldots, \nu_{m^{\prime}-m+s+1}(y)$ est une famille libre d'un supplémentaire de $\mu(y)(\mathfrak{m})$ pour tout $y$ dans $Y^{\prime} \backslash X_{\mathrm{r}}$.

(ii) Soient $e_{1}, \ldots, e_{m}$ une base de $\mathfrak{m}$ et l'application rationnelle

$$
\varepsilon: Y \longrightarrow \wedge^{m}(F), \quad x \longmapsto q(x)^{-s} \mu(x)\left(e_{1}\right) \wedge \cdots \wedge \mu(x)\left(e_{m}\right) .
$$

On suppose que $\varepsilon$ est une application régulière de $Y$ dans $\wedge^{m}(F)$ qui ne s'annule pas sur $Y$. Puisque $E(x)$ contient $\alpha_{\mu}(x)$ pour tout $x$ dans $Y, s$ est la dimension de l'intersection $\mathfrak{m}(x)$ de $E(x)$ et de $\mathfrak{m}$ pour tout $x$ dans $Y \backslash X_{\mathrm{r}}$. En diminuant $Y$ au besoin et en réordonnant la famille $e_{1}, \ldots, e_{m}$, on peut supposer qu'il existe des applications régulières $\varepsilon_{m-s+1}, \ldots, \varepsilon_{m}$ de $Y$ dans $\mathfrak{m}$ qui satisfont la condition suivante : pour tout $x$ dans $Y$, la famille $e_{1}, \ldots, e_{m-s}, \varepsilon_{m-s+1}(x), \ldots, \varepsilon_{m}(x)$ est une base de $\mathfrak{m}$ et pour tout $x$ dans $Y \backslash X_{\mathrm{r}}$, la famille $\varepsilon_{m-s+1}(x), \ldots, \varepsilon_{m}(x)$ est une base de $\mathfrak{m}(x)$. Puisque $q$ engendre l'idéal de définition de $Y \backslash X_{\mathrm{r}}$ dans $\mathbb{C}[Y]$, pour $i=1, \ldots, s$, il existe un entier strictement positif $\ell_{i}$ et une application régulière $\nu_{i}$ de $Y$ dans $F$, non identiquement nulle sur $Y \backslash X_{\mathrm{r}}$, qui satisfont l'égalité

$$
\mu(x)\left(\varepsilon_{m-s+i}(x)\right)=q(x)^{\ell_{i}} \nu_{i}(x),
$$

pour tout $x$ dans $Y$. Puisque $e_{1}, \ldots, e_{m-s}, \varepsilon_{m-s+1}(x), \ldots, \varepsilon_{m}(x)$ est une base de $\mathfrak{m}$ pour tout $x$ dans $Y$, il existe une fonction régulière inversible $a$ sur $Y$ qui

TOME $132-2004-\mathrm{N}^{\mathrm{O}} 4$ 
satisfait l'égalité

$$
e_{1} \wedge \cdots \wedge e_{m-s} \wedge \varepsilon_{m-s+1}(x) \wedge \cdots \wedge \varepsilon_{m}(x)=a(x) e_{1} \wedge \cdots \wedge e_{m},
$$

pour tout $x$ dans $Y$. Par suite, il vient

$$
\begin{array}{r}
\mu(x)\left(e_{1}\right) \wedge \cdots \wedge \mu(x)\left(e_{m-s}\right) \wedge \mu(x)\left(\varepsilon_{m-s+1}(x)\right) \wedge \cdots \wedge \mu(x)\left(\varepsilon_{m}(x)\right) \\
=q(x)^{s} a(x) \varepsilon(x)
\end{array}
$$

pour tout $x$ dans $Y$; donc d'après la régularité de l'application $\varepsilon$ les entiers $\ell_{1}, \ldots, \ell_{s}$ sont égaux à 1 car ils sont tous strictement positifs et pour tout $x$ dans $Y, \mu(x)\left(e_{1}\right), \ldots, \mu(x)\left(e_{m-s}\right), \nu_{1}(x), \ldots, \nu_{s}(x)$ sont linéairement indépendants. Par suite, les applications $e_{1}, \ldots, e_{m-s}, \varepsilon_{m-s+1}, \ldots, \varepsilon_{m}$ de $Y$ dans $E$ et les applications $\nu_{1}, \ldots, \nu_{s}$ de $Y$ dans $F$ satisfont les conditions 1), 2), 3) de la définition 2.4 car $\mu(x)(\mathfrak{m})$ est égal à $x \cdot E$ pour tout $x$ dans $Y$. Cela revient à dire que $Y$ est adéquat.

Réciproquement, on suppose que $Y$ contient un ouvert $Y^{\prime}$ qui rencontre $Y \backslash X_{\mathrm{r}}$ et qui est adéquat. Soient $\varepsilon_{1}, \ldots, \varepsilon_{m}$ des applications régulières de $Y^{\prime}$ dans $E$ et $\nu_{1}, \ldots, \nu_{s}$ des applications régulières de $Y^{\prime}$ dans $F$ qui satisfont les conditions 1), 2), 3) de la définition 2.4 et la condition de l'assertion (i). Alors il existe une fonction régulière inversible $a$ sur $Y^{\prime}$ qui satisfait l'égalité

$$
e_{1} \wedge \cdots \wedge e_{m}=a(x) \varepsilon_{1}(x) \wedge \cdots \wedge \varepsilon_{m}(x)
$$

pour tout $x$ dans $Y^{\prime}$. Par suite, il vient

$$
\begin{aligned}
& \mu(x)\left(e_{1}\right) \wedge \cdots \wedge \mu(x)\left(e_{m}\right)=a(x) \mu(x)\left(\varepsilon_{1}(x)\right) \wedge \cdots \wedge \mu(x)\left(\varepsilon_{m}(x)\right) \\
& \quad=a(x) q(x)^{s} \mu(x)\left(\varepsilon_{1}(x)\right) \wedge \cdots \wedge \mu(x)\left(\varepsilon_{m-s}(x)\right) \wedge \nu_{1}(x) \wedge \cdots \wedge \nu_{s}(x),
\end{aligned}
$$

pour tout $x$ dans $Y^{\prime}$. Il en résulte que l'application rationnelle $\varepsilon$ de $Y$ dans $\wedge^{m}(F)$

$$
x \longmapsto q(x)^{-s} \mu(x)\left(e_{1}\right) \wedge \cdots \wedge \mu(x)\left(e_{m}\right),
$$

a un prolongement régulier à $Y^{\prime}$ qui ne s'annule pas sur $Y^{\prime}$; or la restriction de $\varepsilon$ à l'intersection de $Y^{\prime}$ et de $X_{\mathrm{r}}$ est une application régulière; donc l'application $\varepsilon$ est régulière sur un grand ouvert de $Y$. La variété $Y$ est normale car elle est lisse; donc $\varepsilon$ est une application régulière.

Proposition 2.6. - La restriction de $\mu$ à $Y$ a la propriété $(\mathbf{P})$ si et seulement si pour toute base $e_{1}, \ldots, e_{m}$ de $\mathfrak{m}$, l'application rationnelle de $Y$ dans $\wedge^{m}(F)$

$$
x \longmapsto q(x)^{-s} \mu(x)\left(e_{1}\right) \wedge \cdots \wedge \mu(x)\left(e_{m}\right),
$$

est une application régulière qui n'est pas identiquement nulle sur $Y \backslash X_{\mathrm{r}}$.

Démonstration. - On suppose que la restriction de $\mu$ à $Y$ a la propriété $(\mathbf{P})$. D'après le lemme 2.5, il s'agit de montrer que $Y$ contient un ouvert adéquat qui rencontre $Y \backslash X_{\mathrm{r}}$. On utilise les notations de la démonstration du lemme 2.5. On peut alors supposer qu'il existe des applications régulières $\varepsilon_{m-s+1}, \ldots, \varepsilon_{m}$ de $Y$ dans $\mathfrak{m}$ qui satisfont la condition suivante : pour tout $x$ dans $Y, e_{1}, \ldots, e_{m-s}$, 
$\varepsilon_{m-s+1}(x), \ldots, \varepsilon_{m}(x)$ est une base de $\mathfrak{m}$ et pour tout $x$ dans $Y \backslash X_{\mathrm{r}}$, la famille $\varepsilon_{m-s+1}(x), \ldots, \varepsilon_{m}(x)$ est une base de $\mathfrak{m}(x)$. En outre, pour $i=1, \ldots, s$, il existe un entier strictement positif $\ell_{i}$ et une application régulière $\nu_{i}$ de $Y$ dans $F$, non identiquement nulle sur $Y \backslash X_{\mathrm{r}}$, qui satisfont l'égalité

$$
\mu(x)\left(\varepsilon_{m-s+i}(x)\right)=q(x)^{\ell_{i}} \nu_{i}(x),
$$

pour tout $x$ dans $Y$. Soit $i=1, \ldots, s$. Puisque $q$ est nul sur $Y \backslash X_{\mathrm{r}}, q \nu_{i}$ est section de $\mathcal{M}_{\mu}$ au-dessus de $Y$; donc $q \nu_{i}$ est l'image par $\theta$ d'une application régulière $\psi_{i}$ de $Y$ dans $\mathfrak{m}$ car $\mathfrak{m}$ est un supplémentaire dans $E$ du sous-espace $\alpha_{\mu}(x)$ de $E(x)$ pour tout $x$ dans $Y$. Par suite, $\varepsilon_{m-s+i}$ est égal à $q^{\ell_{i}-1} \psi_{i}$. L'application $\varepsilon_{m-s+i}$ ne s'annulant pas sur $Y, \ell_{i}$ est égal à 1 . On conclut alors comme dans la démonstration de l'assertion (ii) du lemme 2.5.

Réciproquement, on suppose que $\varepsilon$ a un prolongement régulier à $Y$ qui n'est pas identiquement nul sur $Y \backslash X_{\mathrm{r}}$. Alors $Y$ contient un ouvert qui rencontre $X \backslash X_{\mathrm{r}}$ et qui est adéquat d'après l'assertion (ii) du lemme 2.5; or d'après le lemme 2.3, la restriction de $\mu$ à $Y$ a la propriété $(\mathbf{P})$ si $\mathcal{M}_{\mu}$ et l'image de $\theta$ ont même restriction à un grand ouvert de $Y$; donc on peut supposer que $Y$ est un ouvert adéquat. On note $Y_{\mathrm{r}}$ l'intersection de $Y$ et de $X_{\mathrm{r}}, \varepsilon_{1}, \ldots, \varepsilon_{m}$ des applications régulières de $Y$ dans $E$ et $\nu_{1}, \ldots, \nu_{s}$ des applications régulières de $Y$ dans $F$ qui satisfont les conditions 1), 2), 3) de la défintion 2.4 et la condition de l'assertion (i) du lemme 2.5. Soit $\varphi$ une section de $\mathcal{M}_{\mu}$ au-dessus de $Y$. Puisque $\mathfrak{m}$ est un supplémentaire de $E(x)$ dans $E$ pour tout $x$ dans $Y_{\mathrm{r}}$, il existe une application régulière $\psi$ et une seule de $Y_{\mathrm{r}}$ dans $\mathfrak{m}$ qui satisfait l'égalité

$$
\varphi(x)=\mu(x)(\psi(x)),
$$

pour tout $x$ dans $Y_{\mathrm{r}}$. Il s'agit de montrer que $\psi$ a un prolongement régulier à $Y$. Pour tout $x$ dans $Y$, on note $\beta(x)$ le sous-espace de $F$ engendré par les éléments

$$
\mu(x)\left(\varepsilon_{1}(x)\right), \ldots, \mu(x)\left(\varepsilon_{m-s}(x)\right), \nu_{1}(x), \ldots, \nu_{s}(x) .
$$

L'application $\beta$ ainsi définie est une application régulière de $Y$ dans $\operatorname{Gr}_{m}(F)$. En outre, pour tout $x$ dans $Y_{\mathrm{r}}, \beta(x)$ est égal à $x \cdot E$. Par continuité, $\beta(x)$ contient donc $\varphi(x)$ pour tout $x$ dans $Y$. Par suite, il existe des fonctions régulières $a_{1}, \ldots, a_{m}$ sur $Y$ qui satisfont l'égalité

$$
\begin{array}{r}
\varphi(x)=a_{1}(x) \mu(x)\left(\varepsilon_{1}(x)\right)+\cdots+a_{m-s}(x) \mu(x)\left(\varepsilon_{m-s}(x)\right) \\
+a_{m-s+1}(x) \nu_{1}(x)+\cdots+a_{m}(x) \nu_{s}(x),
\end{array}
$$

pour tout $x$ dans $Y$. Pour tout $x$ dans $Y \backslash X_{\mathrm{r}}, \mu(x)\left(\varepsilon_{1}(x)\right), \ldots, \mu(x)\left(\varepsilon_{m-s}(x)\right)$ est une base de $x \cdot E$; donc les fonctions $a_{m-s+1}, \ldots, a_{m}$ sont nulles sur $Y \backslash X_{\mathrm{r}}$. Par suite, il existe des fonctions régulières $b_{1}, \ldots, b_{s}$ sur $Y$ qui satisfont les égalités

$$
a_{m-s+1}=q b_{1}, \ldots, a_{m}=q b_{s} .
$$

TOME $132-2004-\mathrm{N}^{\mathrm{O}} 4$ 
On rappelle que $q$ est un générateur de l'idéal de définition de $Y \backslash X_{\mathrm{r}}$ dans $\mathbb{C}[Y]$. Alors d'après la condition 1 ) de la défintion 2.4 , l'application régulière $\psi^{\prime}$ de $Y$ dans $\mathfrak{m}$

$$
\begin{aligned}
x \longmapsto a_{1}(x) & \varepsilon_{1}(x)+\cdots+a_{m-s}(x) \varepsilon_{m-s}(x) \\
& +b_{1}(x) \varepsilon_{m-s+1}(x)+\cdots+b_{s}(x) \varepsilon_{m}(x),
\end{aligned}
$$

satisfait l'égalité $\varphi(x)=\mu(x)\left(\psi^{\prime}(x)\right.$, pour tout $x$ dans $Y$. D'après l'unicité de $\psi$, l'application $\psi^{\prime}$ est un prolongement de $\psi$ à $Y$.

DÉfinition 2.7. - Un ouvert affine de $X$ est dit admissible pour $\mu$ s'il est contenu dans $X_{\mathrm{r}}$ ou s'il satisfait les conditions 1), 2), 3) du lemme 2.3 et les conditions de la proposition 2.6.

Un ouvert affine $Z$ de $X$ est dit adéquat pour $\mu$ s'il est contenu dans $X_{\mathrm{r}}$ ou s'il satisfait les conditions 1), 2), 3) du lemme 2.3 et est adéquat pour $\mu$ au sens de la définition 2.4 .

Corollaire 2.8. - La réunion des ouverts admissibles pour $\mu$ est un grand ouvert de $X$ si et seulement si la réunion des ouverts adéquats pour $\mu$ est un grand ouvert de $X$.

Démonstration. - Le corollaire résulte de l'assertion (i) du lemme 2.3 et de l'assertion (ii) du lemme 2.5.

ThÉORÈme 2.9. - L'application $\mu$ a la propriété $(\mathbf{P})$ si et seulement si la réunion des ouverts admissibles de $X$ est un grand ouvert de $X$.

Démonstration. - Le théorème résulte de la proposition 2.6 et de l'assertion (ii) du lemme 2.3.

2.3. Pour tout entier strictement positif $r, \mathrm{~S}^{r}(F)$ désigne l'espace des éléments homogènes de degré $r$ de $\mathrm{S}(F)$ pour la structure homogène usuelle. On note $\mu_{r}$ l'application régulière

$$
X \longrightarrow \operatorname{Lin}\left(E \otimes_{\mathbb{C}} \mathrm{S}^{r-1}(F), \mathrm{S}^{r}(F)\right), \quad x \longmapsto(v \otimes w \mapsto \mu(x)(v) w) .
$$

Lemme 2.10. - On suppose que $\mu$ a la propriété $(\mathbf{P})$. Alors pour tout entier strictement positif $r, \mu_{r}$ a la propriété $(\mathbf{P})$.

Démonstration. - D'après le théorème 2.9, le corollaire 2.8 et le lemme 2.3, il suffit de montrer que $X$ est adéquat pour $\mu_{r}$ dans le cas où $X$ est affine, égal à l'ouvert $X_{*}$ et adéquat pour $\mu$. On suppose que $X$ satisfait ces trois conditions. Il existe alors des applications régulières $\varepsilon_{1}, \ldots, \varepsilon_{m}$ de $X$ dans $E$ et des applications régulières $\nu_{1}, \ldots, \nu_{s}$ de $X$ dans $F$ qui satisfont les conditions 1), 2), 3) de la définition 2.4 et la condition de l'assertion (i) du lemme 2.5. Pour tout $x$ dans $X$, on note $\beta(x)$ le sous-espace de $F$ engendré par les éléments

$$
\mu(x)\left(\varepsilon_{1}(x)\right), \ldots, \mu(x)\left(\varepsilon_{m-s}(x)\right), \quad \nu_{1}(x), \ldots, \nu_{s}(x) .
$$

BULletin DE LA SOCiÉtÉ MATHÉMATIQUE DE FRANCE 
Alors $\beta$ ainsi défini est une application régulière de $X$ dans $\operatorname{Gr}_{m}(F)$. Si $x$ est dans $X$ et si $v_{1}, \ldots, v_{d}$ est une base d'un supplémentaire de $\beta(x)$ dans $F$, pour tout $y$ dans un ouvert de $X$, contenant $x, v_{1}, \ldots, v_{d}$ est une base d'un supplémentaire de $\beta(y)$ dans $F$; donc on peut supposer qu'il en est ainsi pour tout $x$ dans $X$.

Pour $j=0, \ldots, r-1$, on désigne par $F_{j, r}$ le sous-espace des éléments de $\mathrm{S}^{r}(F)$ qui sont homogènes de degré $j$ en $v_{1}, \ldots, v_{d}$. Alors la somme des sous-espaces $F_{0, r}, \ldots, F_{r-1, r}$ est directe et contient $\mu_{r}(x)\left(E \otimes_{\mathbb{C}} \mathrm{S}^{r-1}(F)\right)$ pour tout $x$ dans $X$. Soit $\mathfrak{I}_{j}$ l'ensemble des suites croissantes $\iota$ d'entiers $i_{1}, \ldots, i_{f}$ de $\{1, \ldots, d\}$ de somme $j$. Pour $j=0, \mathfrak{I}_{j}$ est vide et le produit $v_{i_{1}} \cdots v_{i_{f}}$ est égal à 1 . Soient

- $\mathfrak{K}_{j}^{\prime}$ l'ensemble des familles $k_{1}, \ldots, k_{e}, \ell_{1}, \ldots, \ell_{e^{\prime}}$ d'entiers naturels qui satisfont les relations

$$
\begin{gathered}
1 \leq k_{1} \leq \cdots \leq k_{e} \leq m-s, \quad 1 \leq \ell_{1} \leq \cdots \leq \ell_{e^{\prime}} \leq s, \\
k_{1}+\cdots+k_{e}+\ell_{1}+\cdots+\ell_{e^{\prime}}=r-j,
\end{gathered}
$$

- $\mathfrak{K}_{j}^{\prime \prime}$ l'ensemble des familles $k_{1}, \ldots, k_{e}$ d'entiers naturels qui satisfont les relations

$$
1 \leq k_{1} \leq \cdots \leq k_{e} \leq m-s, \quad k_{1}+\cdots+k_{e}=r-j,
$$

- $\mathfrak{K}_{j}^{\prime \prime \prime}$ l'ensemble des familles $\ell_{1}, \ldots, \ell_{e^{\prime}}$ d'entiers naturels qui satisfont les relations

$$
1 \leq \ell_{1} \leq \cdots \leq \ell_{e^{\prime}} \leq s, \quad \ell_{1}+\cdots+\ell_{e^{\prime}}=r-j .
$$

On désigne par $\mathfrak{K}_{j}$ la réunion des ensembles $\mathfrak{K}_{j}^{\prime}, \mathfrak{K}_{j}^{\prime \prime}, \mathfrak{K}_{j}^{\prime \prime \prime}$ et par $\tilde{\mathfrak{K}}_{j}$ la réunion des ensembles $\mathfrak{K}_{j}^{\prime}, \mathfrak{K}_{j}^{\prime \prime}$. Pour tout $\kappa$ dans $\mathfrak{K}_{j}^{\prime}$ et pour tout $\iota$ dans $\mathfrak{I}_{j}$, on note $\varepsilon_{\kappa, \iota}$ l'application de $X$ dans $E \otimes_{\mathbb{C}} \mathrm{S}^{r-1}(F)$,

$$
x \longmapsto \varepsilon_{k_{e}}(x) \otimes \mu(x)\left(\varepsilon_{k_{1}}(x)\right) \cdots \mu(x)\left(\varepsilon_{k_{e-1}}(x)\right) \nu_{\ell_{1}}(x) \cdots \nu_{\ell_{e^{\prime}}}(x) v_{i_{1}} \cdots v_{i_{f}} .
$$

Pour tout $\kappa$ dans $\mathfrak{K}_{j}^{\prime \prime}$ et pour tout $\iota$ dans $\mathfrak{I}_{j}$, on note $\varepsilon_{\kappa, \iota}$ l'application de $X$ dans $E \otimes_{\mathbb{C}} \mathrm{S}^{r-1}(F)$,

$$
x \longmapsto \varepsilon_{k_{e}}(x) \otimes \mu(x)\left(\varepsilon_{k_{1}}(x)\right) \cdots \mu(x)\left(\varepsilon_{k_{e-1}}(x)\right) v_{i_{1}} \cdots v_{i_{f}} .
$$

Pour tout $\kappa$ dans $\mathfrak{K}_{j}^{\prime \prime \prime}$ et pour tout $\iota$ dans $\mathfrak{I}_{j}$, on note $\nu_{\kappa, \iota}$ l'application de $X$ dans $\mathrm{S}^{r}(F)$,

et $\varepsilon_{\kappa, \iota}$ l'application de $X$ dans $E \otimes_{\mathbb{C}} \mathrm{S}^{r-1}(F)$

$$
x \longmapsto \nu_{\ell_{1}}(x) \cdots \nu_{\ell_{e^{\prime}}}(x) v_{i_{1}} \cdots v_{i_{f}}
$$

$$
x \longmapsto \varepsilon_{\ell_{e^{\prime}}}(x) \otimes \nu_{\ell_{1}}(x) \cdots \nu_{\ell_{e^{\prime}-1}}(x) v_{i_{1}} \cdots v_{i_{f}} .
$$

Pour tout $(\kappa, \iota)$ dans $\mathfrak{K}_{j} \times \mathfrak{I}_{j}$, les applications $\varepsilon_{\kappa, \iota}$ sont régulières et pour tout $(\kappa, \iota)$ dans $\mathfrak{K}_{j}^{\prime \prime \prime} \times \mathfrak{I}_{j}$, les applications $\nu_{\kappa, \iota}$ sont régulières. En outre, ces applications satisfont les conditions suivantes pour $j=0, \ldots, r-1$ :

A) pour tout $x$ dans $X$, pour tout $\kappa$ dans $\mathfrak{K}_{j}^{\prime \prime \prime}$ et pour tout $\iota$ dans $\mathfrak{I}_{j}$, on a

$$
\mu_{r}(x)\left(\varepsilon_{\kappa, \iota}(x)\right)=q(x) \nu_{\kappa, \iota}(x)
$$

TOME $132-2004-\mathrm{N}^{\mathrm{O}} 4$ 
B) pour tout $x$ dans $X$, la famille suivante est une base de $F_{j, r}$ :

$$
\nu_{\kappa, \iota}(x), \kappa \in \mathfrak{K}_{j}^{\prime \prime \prime}, \iota \in \mathfrak{I}_{j}, \quad \mu_{r}(x)\left(\varepsilon_{\kappa, \iota}(x)\right), \kappa \in \tilde{\mathfrak{K}}_{j}, \iota \in \mathfrak{I}_{j} .
$$

Soit $x$ dans $X \backslash X_{\mathrm{r}}$. Puisque la famille $\nu_{1}(x), \ldots, \nu_{s}(x), v_{1}, \ldots, v_{d}$ est une base d'un supplémentaire de $x \cdot E$ dans $F$, la famille

$$
\mu_{r}(x)\left(\varepsilon_{\kappa, \iota}(x)\right), \quad \kappa \in \tilde{\mathfrak{K}}_{j}, \iota \in \mathfrak{I}_{j}, j=0, \ldots, r-1,
$$

est une base de $\mu_{r}(x)\left(E \otimes_{\mathbb{C}} \mathrm{S}^{r-1}(F)\right)$ car on a une somme directe de sousespaces $F_{0, r}, \ldots, F_{r-1, r}$. Les applications $\varepsilon_{\kappa, \iota}$ et $\nu_{\kappa, \iota}$ satisfont donc les conditions $1), 2), 3$ ) de la définition 2.4 relativement à $\mu_{r}$. D'après les conditions $\mathrm{A}$ ) et $\mathrm{B}$ ), la dimension de $\mu_{r}(x)\left(E \otimes_{\mathbb{C}} \mathrm{S}^{r-1}(F)\right)$ ne dépend pas du point $x$ de $X_{\mathrm{r}}$. En outre, la dimension de $\mu_{r}(x)\left(E \otimes_{\mathbb{C}} \mathrm{S}^{r-1}(F)\right)$ ne dépend pas du point $x$ de $X \backslash X_{\mathrm{r}}$; or $X \backslash X_{\mathrm{r}}$ est un diviseur premier de $X$ dont l'idéal de définition dans $\mathbb{C}[X]$ est engendré par $q$ car $X$ est adéquat pour $\mu$; donc $X$ est un ouvert adéquat pour $\mu_{r}$.

\section{Les propriétés $(P)$ et $(R)$.}

Sous deux hypothèses techniques portant sur $\mu$, on donne dans cette section une propriété équivalente à la propriété $(\mathbf{P})$ plus simple que celle obtenue dans la section précédente. On commence par quelques définitions et remarques.

3.1. Conformément à l'usage, on désigne par Ker $\alpha$ le noyau d'un endomorphisme linéaire $\alpha$. Pour tout point $x$ de $X$, on note $\bar{\mu}_{x}$ l'application de $X$ dans $\operatorname{Lin}(E(x), F /(x \cdot E))$ qui à $y$ associe l'application linéaire $v \mapsto \mu(y)(v)+x \cdot E$.

DÉfinition 3.1. - On dira que :

- $\mu$ a la propriété $(\mathbf{R})$ en $x$ si l'indice de $\bar{\mu}_{x}$ est inférieur à l'indice de $\mu$;

- $\mu$ a la propriété $(\mathbf{R})$ si $\mu$ a la propriété $(\mathbf{R})$ en tout point $x$ de $X$.

LEMME 3.2. - Soient $x$ un point de $X$ et $s$ l'entier $\operatorname{dim} E(x)-\mathrm{i}_{\mu}$.

(i) Soit $y$ un point de $X$. Alors on a

$$
\operatorname{dim} \operatorname{Ker} \bar{\mu}_{x}(y)=\operatorname{dim} E(x) \cap E(y)+\operatorname{dim}(x \cdot E) \cap \mu(y)(E(x)) .
$$

(ii) Soit $y$ un point de $X$. Les points $x$ et $y$ satisfont l'inégalité

$$
\operatorname{dim} E(x) \cap E(y)+\operatorname{dim}(x \cdot E) \cap \mu(y)(E(x)) \leq \mathrm{i}_{\mu},
$$

si et seulement s'il existe des éléments $e_{1}, \ldots, e_{s}$ de $E(x)$ pour lesquels la famille $\mu(y)\left(e_{1}\right), \ldots, \mu(y)\left(e_{s}\right)$ est une famille libre d'un supplémentaire de $x \cdot E$ dans $F$. En outre, l'ensemble des points $y$ de $X$ qui satisfont cette inégalité est ouvert dans $X$.

(iii) L'application $\mu$ a la propriété $(\mathbf{R})$ en $x$ si et seulement s'il existe un point $y$ de $X$ qui satisfait l'inégalité

$$
\operatorname{dim} E(x) \cap E(y)+\operatorname{dim}(x \cdot E) \cap \mu(y)(E(x)) \leq \mathrm{i}_{\mu} .
$$

BULletin DE LA SOCiÉtÉ MATHÉMATiQUE DE FRANCE 
Démonstration. - (i) D'après le théorème du rang pour la restriction de $\mu(y)$ à $E(x)$, on a $\operatorname{dim} E(x)=\operatorname{dim} E(x) \cap E(y)+\operatorname{dim} \mu(y)(E(x))$, et d'après le théorème du rang pour $\bar{\mu}_{x}$, on a

$$
\begin{aligned}
\operatorname{dim} E(x)=\operatorname{dim} & \operatorname{Ker} \bar{\mu}_{x}(y) \\
& +\operatorname{dim} \mu(y)(E(x))-\operatorname{dim}(x \cdot E) \cap \mu(y)(E(x)) .
\end{aligned}
$$

Par suite, il vient $\operatorname{dim} \operatorname{Ker} \bar{\mu}_{x}(y)=\operatorname{dim} E(x) \cap E(y)+\operatorname{dim}(x \cdot E) \cap \mu(y)(E(x))$.

(ii) Il existe des éléments $e_{1}, \ldots, e_{s}$ de $E(x)$ pour lesquels la famille $\mu(y)\left(e_{1}\right), \ldots, \mu(y)\left(e_{s}\right)$ est une famille libre d'un supplémentaire de $x \cdot E$ dans $F$ si et seulement si la dimension de l'image de $\bar{\mu}_{x}(y)$ est supérieure à $s$; or la dimension de l'image de $\bar{\mu}_{x}(y)$ est supérieure à $s$ si et seulement si la dimension de $\operatorname{Ker} \bar{\mu}_{x}(y)$ est inférieure à $\mathrm{i}_{\mu}$; donc l'assertion résulte de l'assertion (i). Il résulte du lemme d'algèbre linéaire rappelé après la définition 2.1 et de l'assertion (i) que l'ensemble des points $y$ de $X$ qui satisfont l'inégalité de l'assertion est ouvert dans $X$.

(iii) L'assertion résulte de l'assertion (i).

3.2. Le but de cette section est le théorème suivant qui montre l'équivalence des propriétés $(\mathbf{P})$ et $(\mathbf{R})$ sous deux conditions techniques.

THÉORÈmE 3.3. - On suppose que le morphisme $\mu$ a les deux propriétés suivantes :

1) $\mu$ est un morphisme dominant de $X$ dans un sous-espace affine $L$ de $\operatorname{Lin}(E, F)$,

2) pour tout a dans l'image de $\mu$, la réunion des images des applications linéaires tangentes à $\mu$ en les points de chaque composante irréductible de la fibre de $\mu$ en a, est partout dense dans le sous-espace $L_{0}$ des translations de $L$.

Alors $\mu$ a la propriété $(\mathbf{P})$ de la définition 2.2 si et seulement si $\mu$ a la propriété $(\mathbf{R})$ en tout point d'un grand ouvert de $X$.

Le reste de cette section est consacré à la démonstration de ce théorème. Comme $X_{*}$ est un grand ouvert de $X$, il suffit de le montrer pour la restriction de $\mu$ à $X_{*}$. On supposera dans les sous-sections qui suivent que $X$ est égal à $X_{*}$.

3.3. On rappelle que $\alpha_{\mu}$ est une application régulière de $X$ dans $\operatorname{Gr}_{\mathrm{i}_{\mu}}(E)$ pour laquelle $\alpha_{\mu}(x)$ est égal à $E(x)$ pour tout $x$ dans $X_{\mathrm{r}}$.

LEMME 3.4. - (i) La restriction de $\mu$ à tout ouvert non vide $U$ de $X$ satisfait les conditions 1) et 2) du théorème.

(ii) Il existe un recouvrement fini d'un grand ouvert de $X$ par des ouverts affines $X_{1}, \ldots, X_{\ell}$ qui ont les propriétés suivantes :

TOME $132-2004-\mathrm{N}^{\mathrm{O}} 4$ 
(a) $X_{i} \backslash X_{\mathrm{r}}$ est une hypersurface lisse, irréductible dont l'idéal de définition dans $\mathbb{C}\left[X_{i}\right]$ est engendré par un élément $q$,

(b) il existe un sous-espace $\mathfrak{m}$ de $E$ qui est un supplémentaire de $\alpha_{\mu}(x)$ dans $E$ pour tout $x$ dans $X_{i}$,

(c) si $X_{i}$ rencontre $X \backslash X_{\mathrm{r}}$, il existe un sous-espace $\mathfrak{p}$ de $\mathfrak{m}$ qui est un supplémentaire de $E(x)$ dans $E$ pour tout $x$ dans $X_{i} \backslash X_{\mathrm{r}}$.

(iii) Si le théorème 3.3 est vrai pour la restriction de $\mu$ à chacun des ouverts $X_{1}, \ldots, X_{\ell}$, alors il est vrai pour $X$.

(iv) Si $X$ est égal à $X_{\mathrm{r}}, \mu$ a les propriétés $(\mathbf{P})$ et $(\mathbf{R})$.

Démonstration. - (i) Puisque $\mu$ est un morphisme dominant, la restriction de $\mu$ à $U$ est un morphisme dominant car $U$ n'est pas vide. Soient $a$ un point de l'image de la restriction de $\mu$ à $U$ et $Z$ une composante irréductible de la fibre de $\mu$ en $a$ qui rencontre $U$. On désigne par $\Sigma$ l'image réciproque de $Z$ par la projection canonique du fibré tangent à $X \operatorname{sur} X$. D'après la condition 2) du théorème 3.3 , la restriction à $\Sigma$ de l'application linéaire tangente à $\mu$ est un morphisme dominant de $\Sigma$ dans $L_{0}$; or l'ensemble $\Sigma_{U}$ des points de $\Sigma$ au-dessus de $U$ est un ouvert partout dense de $\Sigma$ car $Z$ est une sous-variété irréductible de la fibre de $\mu$ en $a$ qui rencontre $U$; donc la restriction à $\Sigma_{U}$ de l'application linéaire tangente à $\mu$ est un morphisme dominant de $\Sigma_{U}$ dans $L_{0}$. Vu l'arbitraire de la composante irréductible $Z$ de la fibre de $\mu$ en a qui rencontre $U$, la restriction de $\mu$ à $U$ satisfait la condition 2) du théorème 3.3 .

(ii) D'après l'assertion (i) du lemme 2.3, il existe un recouvrement fini d'un grand ouvert de $X$ par des ouverts affines $V$ qui satisfont les conditions (a), (b) et la condition suivante : la dimension de $E(x)$ ne dépend pas du point $x$ de $V \backslash X$ r. Soit $V$ un ouvert affine de $X$ qui satisfait ces tois conditions. Si $V$ ne rencontre pas $X \backslash X_{\mathrm{r}}, V$ satisfait les conditions (a), (b), (c). Si $x$ est un point de $V \backslash X_{\mathrm{r}}$, il existe un sous-espace $\mathfrak{p}$ de $\mathfrak{m}$ qui est un supplémentaire de $E(x)$ dans $E$ car $E(x)$ contient $\alpha_{\mu}(x)$. Alors p est un supplémentaire de $E(y)$ dans $E$ pour tout point $y$ d'un ouvert de $V \backslash X_{\mathrm{r}}$ qui contient $x$; donc $x$ appartient à un ouvert affine de $V$ qui vérifie les conditions (a), (b), (c). Par suite, $V$ admet un recouvrement fini par des ouverts affines qui satisfont les conditions (a), (b), (c).

(iii) $\mathrm{Vu}$ la nature locale de la propriété $(\mathbf{P}), \mu$ a la propriété $(\mathbf{P})$ si et seulement si la restriction de $\mu$ à chacun des ouverts $X_{1}, \ldots, X_{\ell}$ a la propriété $(\mathbf{P})$. Pour $i=1, \ldots, \ell$, on note $X_{i}^{\prime}$ l'ensemble des points de $X_{i}$ en lesquels $\mu$ a la propriété $(\mathbf{R})$. Si $X_{i}^{\prime}$ contient un grand ouvert de $X_{i}$ pour $i=1, \ldots, \ell, \mu$ a la propriété $(\mathbf{R})$ en tout point d'un grand ouvert de $X$. Pour $i=1, \ldots, \ell$, l'intersection de $X_{i}$ et d'un grand ouvert de $X$ est un grand ouvert de $X_{i}$; donc la restriction de $\mu$ à $X_{i}$ a la propriété $(\mathbf{R})$ en tout point d'un grand ouvert de $X_{i}$ si $\mu$ a la propriété $(\mathbf{R})$ en tout point d'un grand ouvert de $X$, d'où l'assertion.

(iv) On suppose $X$ égal à $X_{\mathrm{r}}$. Alors $\mu$ a la propriété $(\mathbf{P})$ et par définition, $\mu$ a la propriété $(\mathbf{R})$ en tout point de $X$.

BULlETIN DE LA SOCIÉTÉ MATHÉMATIQUE DE FRANCE 
Le lemme montre qu'il suffit de montrer le théorème pour $X$ affine, vérifiant (a), (b), (c) et contenant strictement $X_{\mathrm{r}}$. On supposera qu'il en est ainsi dans la suite de cette section.

3.4. On note $e_{1}, \ldots, e_{m}$ une base de $\mathfrak{m}$ dont les $m-s$ premiers éléments engendrent $\mathfrak{p}$ et $\delta$ l'application de $X$ dans $\wedge^{m}(F)$

$$
x \longmapsto \mu(x)\left(e_{1}\right) \wedge \cdots \wedge \mu(x)\left(e_{m}\right) .
$$

Soient $T$ une indéterminée, $\tau$ et $\tau_{0}$ les images respectives de $T$ par les applications canoniques de l'anneau de polynômes $\mathbb{C}[T]$ dans les quotients de $\mathbb{C}[T]$ par les idéaux $T^{s+1} \mathbb{C}[T]$ et $T^{2} \mathbb{C}[T]$. Pour $\nu \mathbb{C}[\tau]$-point de $X$, on note $\gamma_{\nu}$ l'évaluation en $\nu$ de l'anneau $\mathcal{O}_{X, x}$ où $x$ est l'image de $\nu$ par la projection canonique de l'ensemble des $\mathbb{C}[\tau]$-points de $X$ sur l'ensemble des $\mathbb{C}$-points de $X$. Alors $\gamma_{\nu}$ est un morphisme de l'anneau $\mathcal{O}_{X, x}$ dans l'anneau $\mathbb{C}[\tau]$. Pour tout entier naturel $j$, on désigne par $\gamma_{\nu, j}$ le morphisme

$$
\mathcal{O}_{X, x} \otimes_{\mathbb{C}} \wedge^{j}(F) \longrightarrow \mathbb{C}[\tau] \otimes_{\mathbb{C}} \wedge^{j}(F), \quad \varphi \otimes u \longmapsto \gamma_{\nu}(\varphi) \otimes u,
$$

où $\varphi$ est dans $\mathcal{O}_{X, x}$ et où $u$ est dans $\wedge^{j}(F)$.

Lemme 3.5. - Soient $x$ un point de $X \backslash X_{\mathrm{r}}, w^{\prime}$ un vecteur tangent $\grave{a} X$ en $x$, $w$ l'image de $w^{\prime}$ par l'application linéaire tangente à $\mu$ en $x$ et $\nu$ un $\mathbb{C}[\tau]$-point de $X$ au-dessus du $\mathbb{C}\left[\tau_{0}\right]$-point de $X$ défini par $w^{\prime}$.

(i) Le sous-module $\tau^{s} \mathbb{C}[\tau] \otimes_{\mathbb{C}} \wedge^{m}(F)$ de $\mathbb{C}[\tau] \otimes_{\mathbb{C}} \wedge^{m}(F)$ contient $\gamma_{\nu, m}(\delta)$.

(ii) L'image de $\delta$ par $\gamma_{\nu, m}$ est non nulle si et seulement s'il existe des éléments $f_{1}, \ldots, f_{s}$ de $E(x)$ pour lesquels la famille $w\left(f_{1}\right), \ldots, w\left(f_{s}\right)$ est une famille libre d'un supplémentaire de $x \cdot E$ dans $F$.

Démonstration. - Désignant par $e_{1}^{\prime}, \ldots, e_{s}^{\prime}$ les projections respectives de $e_{m-s+1}, \ldots, e_{m}$ sur $E(x)$ parallèlement à $\mathfrak{p}, \delta$ est égal à l'application

$$
y \mapsto \mu(y)\left(e_{1}\right) \wedge \cdots \wedge \mu(y)\left(e_{m-s}\right) \wedge \mu(y)\left(e_{1}^{\prime}\right) \wedge \cdots \wedge \mu(y)\left(e_{s}^{\prime}\right) ;
$$

donc on peut supposer que $E(x)$ contient $e_{m-s+1}, \ldots, e_{m}$. Soit $\gamma_{\nu}^{\prime}$ l'application linéaire

$$
\mathcal{O}_{X, x} \otimes_{\mathbb{C}} \operatorname{Lin}(E, F) \longrightarrow \mathbb{C}[\tau] \otimes_{\mathbb{C}} \operatorname{Lin}(E, F), \quad \varphi \otimes a \longmapsto \gamma_{\nu}(\varphi) \otimes a,
$$

où $\varphi$ est dans $\mathcal{O}_{X, x}$ et où $a$ est dans $\operatorname{Lin}(E, F)$. On note $\nu_{2}(\tau)$ l'élément de $\mathbb{C}[\tau] \otimes_{\mathbb{C}} \operatorname{Lin}(E, F)$ défini par la relation

$$
\gamma_{\nu}^{\prime}(\mu)=\mu(x)+\tau w+\tau^{2} \nu_{2}(\tau) .
$$

Alors on a

$$
\begin{aligned}
\gamma_{\nu, m}(\delta)=\left(\mu(x)\left(e_{1}\right)+\right. & \left.\tau w\left(e_{1}\right)+\tau^{2} \nu_{2}(\tau)\left(e_{1}\right)\right) \\
& \wedge \cdots \wedge\left(\mu(x)\left(e_{m}\right)+\tau w\left(e_{m}\right)+\tau^{2} \nu_{2}(\tau)\left(e_{m}\right)\right) .
\end{aligned}
$$

TOME $132-2004-\mathrm{N}^{\mathrm{O}} 4$ 
(i) Puisque $E(x)$ contient $e_{m-s+1}, \ldots, e_{m}$, on a

$$
\gamma_{\nu, m}(\delta)=\tau^{s} \mu(x)\left(e_{1}\right) \wedge \cdots \wedge \mu(x)\left(e_{m-s}\right) \wedge w\left(e_{m-s+1}\right) \wedge \cdots \wedge w\left(e_{m}\right),
$$

d'où l'assertion.

(ii) On suppose qu'il existe des éléments $f_{1}, \ldots, f_{s}$ de $E(x)$ pour lesquels la famille $w\left(f_{1}\right), \ldots, w\left(f_{s}\right)$ est une famille libre d'un supplémentaire de $x \cdot E$ dans $F$. L'intersection de $E(x)$ et de $\mathfrak{m}$ étant de dimension $s, f_{1}, \ldots, f_{s}$ et $e_{m-s+1}, \ldots, e_{m}$ sont des bases du même espace vectoriel; donc $w\left(e_{m-s+1}\right), \ldots, w\left(e_{m}\right)$ est une famille libre d'un supplémentaire de $x \cdot E$ dans $F$ et d'après l'égalité de (i), $\gamma_{\nu, m}(\delta)$ n'est pas nul. Réciproquement, on suppose que $\gamma_{\nu, m}(\delta)$ n'est pas nul. Alors l'ensemble des éléments $\mu(x)\left(e_{1}\right), \ldots, \mu(x)\left(e_{m-s}\right), w\left(e_{m-s+1}\right), \ldots, w\left(e_{m}\right)$ est une partie libre de $F$; or les $m-s$ premiers de ces éléments engendrent $x \cdot E$; donc l'ensemble des éléments $w\left(e_{m-s+1}\right), \ldots, w\left(e_{m}\right)$ est une partie libre d'un supplémentaire de $x \cdot E$ dans $F$.

3.5. Pour tout point $x$ de $X$, on désigne par $\mathrm{T}_{x} X$ l'espace tangent à $X$ en $x$ et par $K_{x}$ le noyau de la différentielle de $q$ en $x$. On note $\Delta$ l'ensemble des points $\left(x, w, f_{1}, \ldots, f_{s}\right)$ de $\left(X \backslash X_{\mathrm{r}}\right) \times L_{0} \times E^{s}$ qui satisfont les conditions suivantes : $E(x)$ contient $f_{1}, \ldots, f_{s},\left\{w\left(f_{1}\right), \ldots, w\left(f_{s}\right)\right\}$ est une partie libre d'un supplémentaire de $x \cdot E$ dans $F$ et $w$ appartient à l'image de $\mathrm{T}_{x} X \backslash K_{x}$ par l'application linéaire tangente à $\mu$ en $x$. Pour $U$ ouvert de $X$, non contenu dans $X_{\mathrm{r}}$, on désigne par $\Delta_{U}$ l'intersection de $U$ et de l'image de $\Delta$ par la projection canonique de $\left(X \backslash X_{\mathrm{r}}\right) \times L_{0} \times E^{s}$ sur $X \backslash X_{\mathrm{r}}$

Lemme 3.6. - Soient $U$ un ouvert de $X$ qui n'est pas contenu dans $X_{\mathrm{r}}$ et $U^{\prime}$ l'ensemble des points de $U$ en lesquels la différentielle de q n'est pas nulle.

(i) La partie $U^{\prime}$ de $X$ est un ouvert qui rencontre $X \backslash X_{\mathrm{r}}$.

(ii) La partie $\Delta_{U^{\prime}}$ de $X \backslash X_{\mathrm{r}}$ est constructible.

(iii) Si $\mu$ a la propriété $(\mathbf{R})$ en tout point d'un ouvert de $X$ qui rencontre $X \backslash X_{\mathrm{r}}, \Delta_{U^{\prime}}$ est partout dense dans $X \backslash X_{\mathrm{r}}$.

(iv) L'application $\mu$ a la propriété $(\mathbf{R})$ en tout point d'un ouvert de $X$ qui rencontre $X \backslash X_{\mathrm{r}}$ si et seulement si $\Delta_{X}$ contient un ouvert non vide de $X \backslash X_{\mathrm{r}}$.

Démonstration. - (i) L'ensemble des points de $X$ en lesquels la différentielle de $q$ n'est pas nulle, est un ouvert de $X$. Cet ouvert rencontre $X \backslash X_{\mathrm{r}}$ car $q$ engendre l'idéal de défintion de $X \backslash X_{\mathrm{r}}$ dans $\mathbb{C}[X]$.

(ii) Soit $\Delta_{1}$ l'ensemble des points $(x, w)$ de $X \times L_{0}$ pour lesquels $w$ est l'image par l'application linéaire tangente à $\mu$ en $x$ d'un élément de $\mathrm{T}_{x} X \backslash K_{x}$. Alors $\Delta_{1}$ est l'image par l'application linéaire tangente à $\mu$ d'un ouvert du fibré tangent à $X$; donc $\Delta_{1}$ est une partie constructible de $X \times L_{0}$. Soient $\Delta_{2}$ l'ensemble des points $\left(x, f_{1}, \ldots, f_{s}\right)$ de $X \times E^{s}$ pour lesquels $E(x)$ contient $f_{1}, \ldots, f_{s}$ et $\Delta_{3}$ l'ensemble des points $\left(x, w, f_{1}, \ldots, f_{s}\right)$ de $X \times L_{0} \times E^{s}$ pour lesquels l'ensemble des éléments $w\left(f_{1}\right), \ldots, w\left(f_{s}\right)$ est une partie libre d'un supplémentaire de $x \cdot E$ 
dans $F$. Si $Y$ est une partie localement fermée de $X$ pour laquelle le rang de $\mu(x)$ ne dépend pas du point $x$ de $Y$, l'intersection de $Y \times E^{s}$ et de $\Delta_{2}$ est fermée dans $Y \times E^{s}$ d'après le lemme d'algèbre linéaire rappelé en 2 . De même, l'intersection de $Y \times L_{0} \times E^{s}$ et de $\Delta_{3}$ est fermée dans $Y \times L_{0} \times E^{s}$; donc $\Delta_{2}$ est une partie constructible de $X \times E^{s}$ et $\Delta_{3}$ est une partie constructible de $X \times L_{0} \times E^{s}$. Désignant par $\Delta_{2}^{\prime}$ l'image réciproque de $\Delta_{2}$ par la projection canonique de $X \times L_{0} \times E^{s}$ sur $X \times E^{s}, \Delta$ est l'intersection des sous-ensembles $\Delta_{1} \times E^{s}, \Delta_{2}^{\prime}, \Delta_{3},\left(X \backslash X_{\mathrm{r}}\right) \times L_{0} \times E^{s}$; donc $\Delta$ est une partie constructible de $\left(X \backslash X_{\mathrm{r}}\right) \times L_{0} \times E^{s}$. Il en résulte que $\Delta_{X}$ et $\Delta_{U^{\prime}}$ sont des parties constructibles de $X \backslash X_{\mathrm{r}}$.

(iii) On suppose que $\mu$ a la propriété $(\mathbf{R})$ en tout point d'un ouvert non vide $W$ de $X \backslash X_{\mathrm{r}}$. Puisque $X \backslash X_{\mathrm{r}}$ est irréductible, l'intersection $V$ de $W$ et de $U^{\prime}$ est un ouvert partout dense de $X \backslash X_{\mathrm{r}}$ d'après l'assertion (i). Soit $x$ dans $V$. D'après les assertions (ii) et (iii) du lemme 3.2, il existe des éléments $f_{1}, \ldots, f_{s}$ de $E(x)$ et un point $y$ de $X$ pour lequel l'ensemble des éléments $\mu(y)\left(f_{1}\right), \ldots, \mu(y)\left(f_{s}\right)$ est une partie libre d'un supplémentaire de $x \cdot E$ dans $F$. Soit $\Omega_{x}$ l'ensemble des éléments $w$ de $L_{0}$ qui satisfont la condition suivante : l'ensemble des éléments $w\left(f_{1}\right), \ldots, w\left(f_{s}\right)$ est une partie libre d'un supplémentaire de $x \cdot E$ dans $F$. Alors $\Omega_{x}$ est ouvert dans $L_{0}$. En outre, $\Omega_{x}$ n'est pas vide car il contient $\mu(y)-\mu(x)$. D'après l'assertion (i) du lemme 3.4 , la restriction $\mu^{\prime}$ de $\mu$ à $U^{\prime}$ satisfait la condition 2 ) du théorème 3.3 ; donc pour tout point $x^{\prime}$ d'un ouvert partout dense $V_{x}$ de la fibre de $\mu^{\prime}$ en $\mu(x)$, l'image de l'application linéaire tangente à $\mu$ en $x^{\prime}$ rencontre $\Omega_{x}$. Il en résulte que pour tout $x^{\prime}$ dans $V_{x}, \Omega_{x}$ rencontre l'image de $\mathrm{T}_{x^{\prime}} X \backslash K_{x^{\prime}}$ par l'application linéaire tangente à $\mu$ en $x^{\prime}$. Puisque $x \cdot E$ et $E(x)$ ne dépendent que de $\mu(x)$, pour tout $x^{\prime}$ dans $V_{x}$, il existe un élément $w$ de $L_{0}$ pour lequel $\Delta$ contient $\left(x^{\prime}, w, f_{1}, \ldots, f_{s}\right)$; donc $\Delta_{X}$ et $\Delta_{U^{\prime}}$ contiennent $V_{x}$. Puisque $V$ est partout dense dans $X \backslash X_{\mathrm{r}}, \Delta_{U^{\prime}}$ est partout dense dans $X \backslash X_{\mathrm{r}}$.

(iv) D'après les assertions (ii) et (iii), $\Delta_{X}$ contient un ouvert non vide de $X \backslash X_{\mathrm{r}}$ si $\mu$ a la propriété $(\mathbf{R})$ en tout point d'un ouvert de $X$ qui rencontre $X \backslash X_{\mathrm{r}}$. Réciproquement, on suppose que $\Delta_{X}$ contient un ouvert non vide $V$ de $X \backslash X_{\mathrm{r}}$. Soit $x$ un point de $V$. Alors il existe des éléments $f_{1}, \ldots, f_{s}$ de $E(x)$ et un élément $w$ de l'image de l'application linéaire tangente à $\mu$ en $x$ qui satisfont la condition suivante : l'ensemble des éléments $w\left(f_{1}\right), \ldots, w\left(f_{s}\right)$ est une partie libre d'un supplémentaire de $x \cdot E$ dans $F$. On note $\Omega_{x}$ l'ensemble des éléments $a$ de $L_{0}$ pour lesquels l'ensemble des éléments $a\left(f_{1}\right), \ldots, a\left(f_{s}\right)$ est une partie libre d'un supplémentaire de $x \cdot E$ dans $F$. Alors $\Omega_{x}$ est un ouvert non vide de $L_{0}$; donc il existe un point $y$ de $X$ pour lequel $\Omega_{x}$ contient $\mu(y)-\mu(x)$ car $\mu$ est un morphisme dominant de $X$ dans $L$. Il en résulte que $\mu$ a la propriété $(\mathbf{R})$ en $x$ d'après les assertions (ii) et (iii) du lemme 3.2.

Démonstration. - On termine la démonstration du théorème 3.3. On utilise les notations des lemmes 3.5 et 3.6. La variété des zéros de $\delta$ contient $X \backslash X_{\mathrm{r}}$

TOME $132-2004-\mathrm{N}^{\mathrm{O}} 4$ 
car $E(x)$ rencontre $\mathfrak{m}$ pour tout point $x$ de $X \backslash X_{\mathrm{r}}$; or $q$ engendre l'idéal de définition de $X \backslash X_{\mathrm{r}}$ dans $\mathbb{C}[X]$; donc $q$ divise $\delta$. Soient $q^{i}$ la plus grande puissance de $q$ qui divise $\delta$ dans $\mathbb{C}[X] \otimes_{\mathbb{C}} \wedge^{m}(F), \delta^{\prime}$ le quotient de $\delta$ par $q^{i}$ et $U$ le complémentaire dans $X$ de la variété des zéros de $\delta^{\prime}$. Alors $U$ est un ouvert de $X$ qui rencontre $X \backslash X_{\mathrm{r}}$. D'après le théorème 2.9 , la propriété $(\mathbf{P})$ pour $\mu$ est équivalente à l'égalité $i=s$. D'après l'assertion (iv) du lemme 3.6, la propriété $(\mathbf{R})$ pour $\mu$ en tout point d'un grand ouvert de $X$ est équivalente à la condition suivante : $\Delta_{X}$ contient un ouvert non vide de $X \backslash X_{\mathrm{r}}$.

D'après l'assertion (i) du lemme 3.6, l'ensemble $U^{\prime}$ des points de $U$ en lesquels la différentielle de $q$ n'est pas nulle, est un ouvert de $X$ qui rencontre $X \backslash X_{\mathrm{r}}$. Soient $x$ un point de $U^{\prime} \backslash X_{\mathrm{r}}, w^{\prime}$ un élément de $\mathrm{T}_{x} X \backslash K_{x}, w$ l'image de $w^{\prime}$ par l'application linéaire tangente à $\mu$ en $x$ et $\nu$ un $\mathbb{C}[\tau]$-point de $X$ au-dessus de $w^{\prime}$. Puisque $w^{\prime}$ n'est pas dans $K_{x}, \gamma_{\nu}(q)$ est le produit de $\tau$ et d'un élément inversible de $\mathbb{C}[\tau]$. De même, $\gamma_{\nu, m}\left(\delta^{\prime}\right)$ n'appartient pas à $\tau \mathbb{C}[\tau] \otimes_{\mathbb{C}} \wedge^{m}(F)$ car $U$ contient $x$; or $\gamma_{\nu, m}(\delta)$ est le produit de $\gamma_{\nu}(q)^{i}$ et de $\gamma_{\nu, m}\left(\delta^{\prime}\right)$; donc $\gamma_{\nu, m}(\delta)$ est le produit de $\tau^{i}$ et d'un élément qui n'appartient pas à $\tau \mathbb{C}[\tau] \otimes_{\mathbb{C}} \wedge^{m}(F)$. D'après l'assertion (ii) du lemme 3.5, $\gamma_{\nu, m}(\delta)$ est un élément non nul de $\tau^{s} \mathbb{C}[\tau] \otimes_{\mathbb{C}} \wedge^{m}(F)$ si et seulement s'il existe des éléments $f_{1}, \ldots, f_{s}$ de $E^{s}$ pour lesquels $\Delta$ contient $\left(x, w, f_{1}, \ldots, f_{s}\right)$; donc $i$ est égal à $s$ si $\Delta_{U^{\prime}}$ contient $x$. Il résulte alors de l'assertion (iii) du lemme 3.6 que $\mu$ a la propriété $(\mathbf{P})$ si $\mu$ a la propriété $(\mathbf{R})$ en tout point d'un grand ouvert de $X$. Réciproquement si $i$ est égal à $s, \Delta_{X}$ contient $U^{\prime}$.

\section{Application de la propriété (R)}

On désigne par $F^{*}$ le dual de $F$ et par $\mathfrak{C}_{\mu}$ l'ensemble des points $\left(x, v^{\prime}\right)$ de $X \times F^{*}$ pour lesquels $x \cdot E$ est contenu dans le noyau de $v^{\prime}$. On rappelle que $\mathrm{S}(F)$ désigne l'algèbre symétrique de $F$. Le faisceau $\mathcal{O}_{X} \otimes_{\mathbb{C}} \mathrm{S}(F)$ est un faisceau cohérent d'algèbres de fonctions régulières sur des ouverts de $X \times F^{*}$. La fonction $\left(x, v^{\prime}\right) \mapsto\left\langle v^{\prime}, \mu(x)(v)\right\rangle$ est section globale de $\mathcal{O}_{X} \otimes_{\mathbb{C}} \mathrm{S}(F)$ pour tout $v$ dans $E$; on la note $\tau_{\mu}(v)$. On montre dans cette section le théorème :

THÉORÈme 4.1. - On suppose que le morphisme $\mu$ a les propriétés 1) et 2) $d u$ théorème 3.3. Soient $\mathcal{I}_{\mu}$ l'idéal de $\mathcal{O}_{X} \otimes_{\mathbb{C}} \mathrm{S}(F)$ engendré par $\tau_{\mu}(E)$ et $\sqrt{\mathcal{I}_{\mu}}$ le radical de $\mathcal{I}_{\mu}$. Soient $\Sigma$ le support dans $X \times F^{*}$ du $\mathcal{O}_{X} \otimes_{\mathbb{C}} \mathrm{S}(F)$-module $\sqrt{\mathcal{I}_{\mu}} / \mathcal{I}_{\mu}$ et $S$ sa projection sur $X$. Alors $S$ est une sous-variété fermée de codimension supérieure à 2 de $X$ si et seulement si $\mu$ a la propriété $(\mathbf{R})$ en tout point d'un grand ouvert de $X$.

Puisque $\mathfrak{C}_{\mu}$ est la variété des zéros de $\mathcal{I}_{\mu}$ dans $X \times V^{*}, \mathfrak{C}_{\mu}$ contient $\Sigma$. Dans ce qui suit, on suppose que le morphisme $\mu$ a les propriétés 1) et 2) du théorème 3.3 . 
4.1. On rappelle que pour tout entier naturel $r, \mathrm{~S}^{r}(F)$ désigne le sous-espace des éléments homogènes de degré $r$ pour la graduation usuelle sur $\mathrm{S}(F)$. Le groupe multiplicatif $\mathbb{C}^{*}$ agit sur $F^{*}$ par multiplication, d'où une action de $\mathbb{C}^{*}$ sur $X \times F^{*}$. L'idéal $\mathcal{I}_{\mu}$ est stable par cette action; donc $\sqrt{\mathcal{I}_{\mu}}$ est stable par cette action. En outre, la graduation naturelle de $\mathrm{S}(F)$ induit sur $\mathcal{O}_{X} \otimes_{\mathbb{C}} \mathrm{S}(F)$ une structure de $\mathcal{O}_{X}$-algèbre graduée. D'après des résultats classiques sur les anneaux gradués, on a alors le lemme :

LEMme 4.2. - Pour tout entier naturel $r$, on désigne par $\mathcal{I}_{r}$ et $\mathcal{J}_{r}$ les intersections respectives de $\mathcal{I}_{\mu}$ et de $\sqrt{\mathcal{I}_{\mu}}$ avec $\mathcal{O}_{X} \otimes_{\mathbb{C}} \mathrm{S}^{r}(F)$.

(i) L'idéal $\mathcal{I}_{\mu}$ est somme directe des sous- $\mathcal{O}_{X}$-modules $\mathcal{I}_{1}, \mathcal{I}_{2}, \ldots$

(ii) L'idéal $\sqrt{\mathcal{I}_{\mu}}$ est somme directe des sous- $\mathcal{O}_{X}$-modules $\mathcal{J}_{1}, \mathcal{J}_{2}, \ldots$

(iii) Pour $\ell$ entier positif assez grand, $\sqrt{\mathcal{I}_{\mu}}$ est le sous-S $(F)$-module de $\mathcal{O}_{X} \otimes_{\mathbb{C}} \mathrm{S}(F)$ engendré par la somme des sous- $\mathcal{O}_{X}$-modules $\mathcal{J}_{1}, \ldots, \mathcal{J}_{\ell}$.

4.2. Pour tout $t$ dans $\mathbb{C}^{*}$, on note $\kappa_{t}$ l'homothétie vectorielle de rapport $t$ de l'espace vectoriel $F^{*}$.

LEMme 4.3. - Soit $\Sigma$ une partie fermée de $X \times F^{*}$ qui est stable par $1_{X} \times \kappa_{t}$ pour tout élément non nul $t$ de $\mathbb{C}$. Alors la projection de $\Sigma$ sur $X$ est fermée dans $X$.

Démonstration. - Puisque chaque composante irréductible de $\Sigma$ est stable par $1_{X} \times \kappa_{t}$ pour tout élément non nul $t$ de $\mathbb{C}$, on peut supposer $\Sigma$ irréductible. Soit $\Sigma^{\prime}$ l'intersection de $\Sigma$ et de $X \times\left(F^{*} \backslash\{0\}\right)$. On note $\Sigma^{*}$ l'image de $\Sigma^{\prime}$ par l'application $\left(x, v^{\prime}\right) \mapsto\left(x, \varpi\left(v^{\prime}\right)\right)$ où $\varpi$ est l'application canonique de $F^{*} \backslash\{0\}$ sur l'espace projectif $\mathbb{P}\left(F^{*}\right)$ de $F^{*}$. D'après l'hypothèse sur $\Sigma, \Sigma^{\prime}$ est l'image réciproque de $\Sigma^{*}$ par cette application ; or $\Sigma^{\prime}$ est fermé dans $X \times\left(F^{*} \backslash\{0\}\right)$, donc $\Sigma^{*}$ est fermé dans $X \times \mathbb{P}\left(F^{*}\right)$. Par suite, la projection de $\Sigma^{\prime}$ sur $X$ est fermée dans $X$. Puisque $\Sigma$ est irréductible, $\Sigma^{\prime}$ est partout dense dans $\Sigma$ quand il est non vide; donc dans ce cas, la projection de $\Sigma$ sur $X$ est égale à la projection de $\Sigma^{\prime}$ sur $X$ car celle-ci est fermée dans $X$, d'où l'assertion. Si $\Sigma^{\prime}$ est vide, $\Sigma$ est égal à $S \times\{0\}$ où $S$ est la projection de $\Sigma$ sur $X$. La partie $S$ de $X$ est fermée car $\Sigma$ est fermé dans $X \times F^{*}$.

4.3. Soit $\ell$ un entier positif assez grand pour que $\sqrt{\mathcal{I}_{\mu}}$ soit le sous-S $(F)$-module engendré par la somme des sous- $\mathcal{O}_{X}$-modules $\mathcal{J}_{1}, \ldots, \mathcal{J}_{\ell}$. L'existence de $\ell$ résulte de l'assertion (iii) du lemme 4.2. Pour tout entier strictement positif $r$, on note $E_{r}$ l'espace vectoriel $E \otimes_{\mathbb{C}} \mathrm{S}^{r-1}(F)$ et $\mu_{r}$ l'application régulière de $X$ dans $\operatorname{Lin}\left(E_{r}, \mathrm{~S}^{r}(F)\right)$ qui à $x$ associe l'application linéaire $v \otimes a \mapsto \mu(x)(v) a$ où $v$ est dans $E$ et où $a$ est dans $\mathrm{S}^{r-1}(F)$. On note $\mathrm{i}_{\mu_{r}}$ l'indice de $\mu_{r}$ et $X_{\mathrm{r}, r}$ l'ensemble des points $x$ de $X$ pour lesquels $\mathrm{i}_{\mu_{r}}$ est la dimension du noyau de $\mu_{r}(x)$. D'après $[5$, ch. VI, th. 1], il existe un grand ouvert $Z$ de $X$ et une application régulière

$$
\alpha: Z \longrightarrow \operatorname{Gr}_{i_{\mu_{1}}}\left(E_{1}\right) \times \cdots \times \operatorname{Gr}_{i_{\mu_{\ell}}}\left(E_{\ell}\right),
$$

TOME $132-2004-\mathrm{N}^{\mathrm{O}} 4$ 
qui satisfait l'égalité

$$
\alpha(x)=\left(E_{1}(x), \ldots, E_{\ell}(x)\right),
$$

si $x$ appartient à l'intersection des ouverts $X_{\mathrm{r}, 1}, \ldots, X_{\mathrm{r}, \ell}$.

LEMME 4.4. - Soit $r$ un entier dans $\{1, \ldots, \ell\}$.

(i) Le $\mathcal{O}_{X}$-module $\mathcal{J}_{r}$ est égal au module $\mathcal{M}_{\mu_{r}}$ défini dans la définition 2.2.

(ii) Si $\mu$ a la propriété $(\mathbf{R})$ en tout point d'un grand ouvert de $X$, alors $\mathcal{I}_{r}$ et $\mathcal{J}_{r}$ ont même restriction à $Z$.

Démonstration. - Le $\mathcal{O}_{X}$-module $\mathcal{O}_{X} \otimes_{\mathbb{C}} \mathrm{S}^{r}(F)$ s'identifie canoniquement au module des germes d'applications régulières de $X$ dans $\mathrm{S}^{r}(F)$.

(i) Soient $V$ un ouvert de $X$ et $\varphi$ une application régulière de $V$ dans $\mathrm{S}^{r}(F)$. Par définition, $\varphi$ est section locale de $\mathcal{M}_{\mu_{r}}$ si et seulement si l'image de $\mu_{r}(x)$ contient $\varphi(x)$ pour tout $x$ dans $V$. L'application $\varphi$ est section locale de $\mathcal{J}_{r}$ si et seulement si $\varphi$ est nul sur l'intersection de $\mathfrak{C}_{\mu}$ et de $V \times F^{*}$. Pour tout $x$ dans $V$, l'idéal de définition dans $\mathrm{S}(F)$ de l'ensemble des points de $\mathfrak{C}_{\mu}$ au-dessus de $x$ est l'idéal engendré par $x \cdot E$; or l'intersection de cet idéal et de $\mathrm{S}^{r}(F)$ est égale à l'image de $\mu_{r}(x)$; donc $\varphi$ est section de $\mathcal{J}_{r}$ au-dessus de $V$ si et seulement si $\varphi$ est section de $\mathcal{M}_{\mu_{r}}$ au-dessus de $V$, d'où l'assertion.

(ii) On suppose que $\mu$ a la propriété $(\mathbf{R})$ en tout point d'un grand ouvert de $X$. Alors $\mu$ a la propriété $(\mathbf{P})$ de la définition 2.2 d'après le théorème 3.3 ; donc d'après le lemme $2.10, \mu_{r}$ a la propriété $(\mathbf{P})$. Il résulte alors de l'assertion (i) que la restriction de $\mathcal{J}_{r}$ à $Z$ est la restriction à $Z$ de l'image du morphisme $\theta_{r}$

$$
\mathcal{O}_{X} \otimes_{\mathbb{C}} E_{r} \longrightarrow \mathcal{O}_{X} \otimes_{\mathbb{C}} \mathrm{S}^{r}(F), \quad \varphi \otimes v \longmapsto\left(x \mapsto \varphi(x) \mu_{r}(x)(v)\right),
$$

où $\varphi$ est section locale de $\mathcal{O}_{X}$ et où $v$ est dans $E_{r}$; or $\mathcal{I}_{r}$ est l'image de $\theta_{r}$ car pour tout $v$ dans $E$ et pour tout $a$ dans $\mathrm{S}^{r-1}(F), \tau(v) a$ est la fonction sur $X \times F^{*},\left(x, v^{\prime}\right) \mapsto \mu_{r}(x)(v \otimes a)\left(v^{\prime}\right)$; donc $\mathcal{I}_{r}$ et $\mathcal{J}_{r}$ ont même restriction à $Z$.

Démonstration du théorème 4.1. — D'après le lemme 4.2, le support $\Sigma \mathrm{du}$ $\mathcal{O}_{X} \otimes_{\mathbb{C}} \mathrm{S}(F)$-module $\sqrt{\mathcal{I}_{\mu}} / \mathcal{I}_{\mu}$ dans $X \times F^{*}$ est stable par l'action de $\mathbb{C}^{*}$; donc d'après le lemme 4.3 , la projection $S$ de $\Sigma$ sur $X$ est fermée dans $X$. On suppose que $\mu$ a la propriété $(\mathbf{R})$ en tout point d'un grand ouvert de $X$. D'après l'assertion (ii) du lemme $4.4, \mathcal{I}_{r}$ et $\mathcal{J}_{r}$ ont même restriction à $Z$ pour $r=1, \ldots, \ell$; or le $\mathrm{S}(F)$-module $\mathcal{J}_{\mu}$ est engendré par la somme des $\mathcal{O}_{X}$-modules $\mathcal{J}_{1}, \ldots, \mathcal{J}_{\ell} ;$ donc $\mathcal{J}_{\mu}$ et $\mathcal{I}_{\mu}$ ont même restriction à $Z \times F^{*}$ car $\mathcal{J}_{\mu}$ contient $\mathcal{I}_{\mu}$ qui est égal au $\mathrm{S}(F)$-module engendré par $\mathcal{I}_{1}$. Il en résulte que $\Sigma$ est contenu dans $(X \backslash Z) \times F^{*}$ et que $X \backslash Z$ contient $S$; donc la codimension de $S$ dans $X$ est supérieure à 2 car $Z$ est un grand ouvert de $X$. Réciproquement, on suppose que $S$ est de codimension supérieure à 2 dans $X$. Puisque $\mathcal{J}_{\mu}$ et $\mathcal{I}_{\mu}$ ont même restriction à $(X \backslash S) \times F^{*}$, les $\mathcal{O}_{X}$-modules $\mathcal{I}_{1}$ et $\mathcal{J}_{1}$ ont même restriction à $X \backslash S$. 
D'après l'assertion (i) du lemme $4.4, \mathcal{J}_{1}$ est égal à $\mathcal{M}_{\mu}$; or $\mathcal{I}_{1}$ est l'image du morphisme $\theta$

$$
\mathcal{O}_{X} \otimes_{\mathbb{C}} E \longrightarrow \mathcal{O}_{X} \otimes_{\mathbb{C}} F, \quad \varphi \otimes v \longmapsto(x \mapsto \varphi(x) \mu(x)(v)),
$$

où $\varphi$ est section locale de $\mathcal{O}_{X}$ et où $v$ est dans $E$; donc d'après l'assertion (ii) du lemme 2.3, $\mu$ a la propriété $(\mathbf{P})$ car $X \backslash S$ est un grand ouvert de $X$. Il résulte alors du théorème 3.3 que $\mu$ a la propriété $(\mathbf{R})$ en tout point d'un grand ouvert de $X$.

\section{Application aux algèbres de Lie}

Soit $\mathfrak{g}$ une algèbre de Lie de dimension finie. On note $\mu$ l'application de $\mathfrak{g}$ dans l'espace des endomorphismes linéaires de $\mathfrak{g}$ qui à $x$ associe ad $x$. On désigne par $\mathfrak{g}^{*}$ le dual de $\mathfrak{g}$ et par $\nu$ l'application de $\mathfrak{g}^{*}$ dans $\operatorname{Lin}\left(\mathfrak{g}, \mathfrak{g}^{*}\right)$ qui à l'élément $v^{\prime}$ de $\mathfrak{g}^{*}$ associe l'application linéaire $\xi \mapsto \xi \cdot v^{\prime}$ où $\xi \cdot v^{\prime}$ est l'action coadjointe de $\xi$ sur $v^{\prime}$.

DÉFinition 5.1. - On dira que l'algèbre de Lie $\mathfrak{g}$ possède :

- la propriété $(\mathbf{C})$ en l'élément $\xi$ de $\mathfrak{g}$ si $\mu$ a la propriété $(\mathbf{R})$ en $\xi$,

- la propriété $(\mathbf{C})$ si $\mu$ a la propriété $(\mathbf{R})$,

- la propriété $(\mathbf{Q})$ en l'élément $v^{\prime}$ de $\mathfrak{g}^{*}$ si $\nu$ a la propriété $(\mathbf{R})$ en $v^{\prime}$,

- la propriété $(\mathbf{Q})$ si $\nu$ a la propriété $(\mathbf{R})$.

5.1. On rappelle qu'une algèbre de Lie $\mathfrak{g}$ est dite quadratique lorsque ses représentations adjointe et coadjointe sont isomorphes et que l'indice $\mathfrak{i}_{\mathfrak{g}}$ de $\mathfrak{g}$ est la dimension minimale des stabilisateurs dans $\mathfrak{g}$ des éléments de $\mathfrak{g}^{*}$. Selon les notations ci-dessus, $i_{\mathfrak{g}}$ est égal à $i_{\nu}$.

Proposition 5.2. - Soit $v^{\prime}$ dans $\mathfrak{g}^{*}$.

(i) L'algèbre de Lie $\mathfrak{g}$ a la propriété $(\mathbf{Q})$ en $v^{\prime}$ si et seulement si l'indice de $\mathfrak{g}\left(v^{\prime}\right)$ est égal à $\mathrm{i}_{\mathfrak{g}}$.

(ii) On suppose $\mathfrak{g}$ quadratique. Alors $\mathfrak{g}$ a la propriété $(\mathbf{C})$ si et seulement si $\mathfrak{g}$ a la propriété $(\mathbf{Q})$.

Démonstration. - (i) Par définition, $\mathfrak{g}$ a la propriété $(\mathbf{Q})$ en $v^{\prime}$ si et seulement si l'indice de $\mathfrak{g}\left(v^{\prime}\right)$ est inférieur à l'indice de $\mathfrak{g}$. L'assertion résulte alors de $[3$, cor. 1.7].

(ii) Il existe par hypothèse un isomorphisme $\sigma$ de $\mathfrak{g}$ sur $\mathfrak{g}^{*}$ qui entrelace les représentations adjointe et coadjointe. En particulier, $\mathrm{i}_{\mu}$ est égal à $\mathrm{i}_{\nu}$. Pour tout $\xi$ dans $\mathfrak{g}, \mathfrak{g}(\xi)$ est égal à $\mathfrak{g}(\sigma(\xi))$ et $\mathfrak{g} \cdot \sigma(\xi)$ est égal à $\sigma(\mathfrak{g} \cdot \xi)$; donc pour tout $\xi$ dans $\mathfrak{g}$, la propriété $(\mathbf{C})$ en $\xi$ est équivalente à la propriété $(\mathbf{Q})$ en $\sigma(\xi)$, d'où l'assertion. 
REMARQUE 5.3. - L'algèbre de Heisenberg a la propriété (Q) mais l'algèbre nilpotente de dimension 5 engendrée par $e_{1}, \ldots, e_{5}$ avec les crochets non nuls :

$$
\left[e_{1}, e_{2}\right]=e_{3}, \quad\left[e_{1}, e_{3}\right]=e_{4}, \quad\left[e_{1}, e_{4}\right]=e_{5}, \quad\left[e_{2}, e_{3}\right]=e_{5},
$$

n'a pas la propriété $(\mathbf{Q})$ en tout point d'une sous-variété de codimension 1 de son dual.

Soit $\mathfrak{C}_{\mathfrak{g}}$ la sous-variété des points $\left(\xi, v^{\prime}\right)$ de $\mathfrak{g} \times \mathfrak{g}^{*}$ pour lesquels $\xi$ stabilise $v^{\prime}$. La variété $\mathfrak{C}_{\mathfrak{g}}$ coïncide avec la variété $\mathfrak{C}_{\mu}$ du théorème 4.1 pour $X=E=F=\mathfrak{g}$. On note $I_{\mathfrak{g}}$ l'idéal de $\mathrm{S}\left(\mathfrak{g}^{*}\right) \otimes_{\mathbb{C}} \mathrm{S}(\mathfrak{g})$ engendré par les fonctions $\left(\xi, v^{\prime}\right) \mapsto\left\langle v^{\prime},[\xi, \eta]\right\rangle$ où $\eta$ est dans $\mathfrak{g}$ et $\sqrt{I_{\mathfrak{g}}}$ son radical.

ThÉORÈme 5.4. - Soit $\Sigma$ le support dans $\mathfrak{g} \times \mathfrak{g}^{*} d u \mathrm{~S}\left(\mathfrak{g}^{*}\right) \otimes_{\mathbb{C}} \mathrm{S}(\mathfrak{g})$-module $\sqrt{I_{\mathfrak{g}}} / I_{\mathfrak{g}}$.

(i) L'algèbre de Lie $\mathfrak{g}$ a la propriété $(\mathbf{C})$ en tout point d'un grand ouvert de $\mathfrak{g}$ si et seulement si la projection de $\Sigma$ sur $\mathfrak{g}$ est une sous-variété fermée de codimension supérieure à 2 de $\mathfrak{g}$.

(ii) L'algèbre de Lie $\mathfrak{g}$ a la propriété $(\mathbf{Q})$ en tout point d'un grand ouvert de $\mathfrak{g}^{*}$ si et seulement si la projection de $\Sigma$ sur $\mathfrak{g}^{*}$ est une sous-variété fermée de codimension supérieure à 2 de $\mathfrak{g}^{*}$.

Démonstration. - (i) Pour $X=E=F=\mathfrak{g}$ et pour $\mu$ égal à l'application $x \mapsto \operatorname{ad} x$ de $\mathfrak{g}$ dans l'espace des endomorphismes linéaires de $\mathfrak{g}, \mathfrak{C}_{\mu}$ est égal à $\mathfrak{C}_{\mathfrak{g}}$. L'application $\mu$ satisfait les conditions 1 ) et 2 ) du théorème 3.3 car $\mu$ est une application linéaire. L'assertion est alors un cas particulier du théorème 4.1.

(ii) Pour $X=F=\mathfrak{g}^{*}, E=\mathfrak{g}$ et pour $\nu$ égal à l'application de $\mathfrak{g}^{*}$ dans $\operatorname{Lin}\left(\mathfrak{g}, \mathfrak{g}^{*}\right)$ qui à l'élément $v^{\prime}$ de $\mathfrak{g}^{*}$ associe l'application linéaire $\xi \mapsto \xi \cdot v^{\prime}$, la restriction à $\mathfrak{C}_{\mathfrak{g}}$ de l'isomorphisme $\left(\xi, v^{\prime}\right) \mapsto\left(v^{\prime}, \xi\right)$ de $\mathfrak{g} \times \mathfrak{g}^{*}$ sur $\mathfrak{g}^{*} \times \mathfrak{g}$ est un isomorphisme de $\mathfrak{C}_{\mathfrak{g}}$ sur $\mathfrak{C}_{\nu}$. En outre, l'image de $I_{\mathfrak{g}}$ par cet isomorphisme est l'espace des sections globales de $\mathcal{I}_{\nu}$. L'application $\nu$ satisfait les conditions 1 ) et 2) du théorème 3.3 car $\nu$ est une application linéaire. L'assertion est alors un cas particulier du théorème 4.1 .

5.2. Dans cette sous-section et les suivantes, on suppose que $\mathfrak{g}$ est une algèbre de Lie réductive. On note $G$ son groupe adjoint. Les représentations adjointe et coadjointe de $\mathfrak{g}$ sont isomorphes. En particulier, l'indice de $\mathfrak{g}$ est égal au rang $\operatorname{rk} \mathfrak{g}$ de $\mathfrak{g}$. Pour tout $x$ dans $\mathfrak{g}, \mathfrak{g}(x)$ désigne le centralisateur de $x$ dans $\mathfrak{g}$.

ThÉORÈme 5.5. - L'indice du centralisateur de tout élément de $\mathfrak{g}$ est égal au rang de $\mathfrak{g}$.

Démonstration. - D'après l'assertion (i) de la proposition 5.2, le théorème revient à dire que l'algèbre de Lie $\mathfrak{g}$ a la propriété $(\mathbf{Q})$. Puisque le centralisateur de tout élément de $\mathfrak{g}$ contient le centre de $\mathfrak{g}$, le théorème pour $\mathfrak{g}$ est conséquence 
du théorème pour $[\mathfrak{g}, \mathfrak{g}]$; donc il suffit de montrer le théorème pour $\mathfrak{g}$ semisimple.

On raisonne par récurrence sur la dimension de $\mathfrak{g}$. Si $\mathfrak{g}$ est simple de dimension 3 , le centralisateur de tout élément non nul de $\mathfrak{g}$ est de dimension 1 ; donc le théorème est vrai pour $\mathfrak{g}$. On suppose le théorème vrai pour toute algèbre de Lie semi-simple de dimension strictement inférieure à dim $\mathfrak{g}$. Soit $x$ un élément non nul de $\mathfrak{g}$. On note $x_{\mathrm{s}}$ et $x_{\mathrm{n}}$ les composantes semi-simple et nilpotente de $x$. Le centralisateur $\mathfrak{g}(x)$ de $x$ est alors égal au centralisateur de $x_{\mathrm{n}}$ dans $\mathfrak{g}\left(x_{\mathrm{s}}\right)$. Si $x_{\mathrm{s}}$ est non nul, $\mathfrak{g}\left(x_{\mathrm{s}}\right)$ est une algèbre de Lie réductive dont l'algèbre dérivée est de dimension strictement inférieure à dim $\mathfrak{g}$; donc d'après l'hypothèse de récurrence, l'indice de $\mathfrak{g}(x)$ est égal au rang de $\mathfrak{g}\left(x_{\mathrm{s}}\right)$. Il en résulte que l'indice de $\mathfrak{g}(x)$ est égal au rang de $\mathfrak{g}$ car $\mathfrak{g}$ et $\mathfrak{g}\left(x_{\mathrm{s}}\right)$ ont même rang. Il s'agit donc de montrer que pour tout élément nilpotent $\xi$ de $\mathfrak{g}$, l'indice de $\mathfrak{g}(\xi)$ est égal au rang de $\mathfrak{g}$. Si $\xi$ est régulier, $\mathfrak{g}(\xi)$ est une sous-algèbre commutative de dimension rk $\mathfrak{g}$ d'après [1, ch. II, 1.8]; donc il suffit de démontrer que l'indice de $\mathfrak{g}(\xi)$ est égal à rk $\mathfrak{g}$ dans le cas où $\xi$ n'est pas régulier.

Le reste de cette section est consacré à la démonstration de cette assertion sous l'hypothèse que le théorème est vrai pour toute algèbre de Lie semi-simple de dimension strictement inférieure à celle de $\mathfrak{g}$.

5.3. Puisque $\xi$ est un élément nilpotent, il résulte du théorème de JacobsonMorozov qu'il existe des éléments $\rho$ et $\eta$ de $\mathfrak{g}$ pour lesquels $\xi, \rho, \eta$ satisfont les relations de $\mathfrak{s l}_{2}$-triplet :

$$
[\xi, \eta]=\rho, \quad[\rho, \xi]=2 \xi, \quad[\rho, \eta]=-2 \eta .
$$

On note $\pi_{\xi}$ l'application $(g, x) \mapsto g(x)$ de $G \times(\xi+\mathfrak{g}(\eta))$ dans $\mathfrak{g}$. On rappelle dans cette sous-section un résultat de P. Slodowy [6]. La notion d'orthogonalité utilisée est celle relative à la forme de Killing de $\mathfrak{g}$.

LEMME 5.6. - L'espace $\mathfrak{g}$ est somme directe des sous-espaces $\mathfrak{g}(\eta)$ et $[\xi, \mathfrak{g}]$. En outre, l'orthogonal de $\mathfrak{g}(\xi)$ dans $\mathfrak{g}$ est égal à $[\xi, \mathfrak{g}]$ et la restriction de la forme de Killing de $\mathfrak{g} \grave{a} \mathfrak{g}(\eta) \times \mathfrak{g}(\xi)$ est non dégénérée.

Démonstration. - Soit $\mathfrak{l}$ le sous-espace de $\mathfrak{g}$ engendré par $\xi, \rho, \eta$. Alors $\mathfrak{l}$ est une sous-algèbre simple de dimension 3 de $\mathfrak{g}$. Il en résulte que $\mathfrak{g}$ est somme directe de sous-l-modules simples $V_{1}, \ldots, V_{p}$. Pour $i=1, \ldots, p$, on désigne par $w_{i}$ un élément non nul de $V_{i}$ qui appartient au noyau de ad $\eta$ et $V_{i}^{\prime}$ l'image de la restriction de ad $\xi$ à $V_{i}$. Alors $V_{i}$ est somme directe de $V_{i}^{\prime}$ et de la droite engendrée par $w_{i}$. Le centralisateur $\mathfrak{g}(\eta)$ de $\eta$ est le sous-espace engendré par $w_{1}, \ldots, w_{p}$ et $[\xi, \mathfrak{g}]$ est somme directe des sous-espaces $V_{1}^{\prime}, \ldots, V_{p}^{\prime} ;$ donc $\mathfrak{g}$ est somme directe de $\mathfrak{g}(\eta)$ et de $[\xi, \mathfrak{g}]$.

D'après la propriété d'invariance de la forme de Killing, $\mathfrak{g}(\xi)$ est orthogonal à $[\xi, \mathfrak{g}]$; or d'après ce qui précède, la dimension de $\mathfrak{g}(\xi)$ est égale à la codimension de $[\xi, \mathfrak{g}]$ dans $\mathfrak{g}$ car $\mathfrak{g}(\xi)$ et $\mathfrak{g}(\eta)$ ont même dimension; donc $\mathfrak{g}(\xi)$ est

TOME $132-2004-\mathrm{N}^{\mathrm{O}} 4$ 
l'orthogonal de $[\xi, \mathfrak{g}]$ dans $\mathfrak{g}$. Puisque l'intersection de $\mathfrak{g}(\eta)$ et de $[\xi, \mathfrak{g}]$ est nulle, l'othogonal de $\mathfrak{g}(\xi)$ dans $\mathfrak{g}(\eta)$ est nul. En permutant les rôles de $\xi$ et de $\eta$, on voit que l'othogonal de $\mathfrak{g}(\eta)$ dans $\mathfrak{g}(\xi)$ est nul, d'où le lemme.

Corollaire 5.7. - Il existe un voisinage ouvert $W$ de $\xi$ dans $\xi+\mathfrak{g}(\eta)$ tel que la restriction de $\pi_{\xi}$ à $G \times W$ soit un morphisme lisse de $G \times W$ sur un ouvert $G$-invariant de $\mathfrak{g}$ qui contient $\xi$.

Démonstration. - D'après le lemme, $\pi_{\xi}$ est une submersion en $(e, \xi)$; donc il existe un voisinage ouvert $W$ de $\xi$ dans $\xi+\mathfrak{g}(\eta)$ tel que $\pi_{\xi}$ soit une submersion en $(e, x)$ pour tout $x$ dans $W$. Si $(g, x)$ est un point de $G \times(\xi+\mathfrak{g}(\eta))$ en lequel $\pi_{\xi}$ est une submersion, $\pi_{\xi}$ est une submersion en $(h g, x)$ pour tout $h$ dans $G$; donc la restriction de $\pi_{\xi}$ à $G \times W$ est une submersion en tout point, d'où le corollaire car l'image de $\pi_{\xi}$ est invariante par $G$.

5.4. On rappelle qu'une forme linéaire $v^{\prime}$ sur $\mathfrak{g}(\xi)$ est dite régulière si son stabilisateur dans $\mathfrak{g}(\xi)$ est de dimension minimale. Si $v^{\prime}$ est une forme linéaire régulière sur $\mathfrak{g}(\xi)$, son stabilisateur $\mathfrak{g}(\xi)\left(v^{\prime}\right)$ dans $\mathfrak{g}(\xi)$ est une sous-algèbre commutative d'après $[1$, ch. II, 1.8$]$; donc l'ensemble $\mathfrak{s}\left(v^{\prime}\right)$ des éléments semi-simples de $\mathfrak{g}(\xi)\left(v^{\prime}\right)$ est un sous-espace de $\mathfrak{g}(\xi)$. Selon M. Duflo, la forme linéaire régulière $v^{\prime}$ est dite fortement régulière $\mathrm{si} \mathfrak{s}\left(v^{\prime}\right)$ est de dimension maximale. L'algèbre de Lie $\mathfrak{g}$ étant semi-simple, la représentation adjointe de $\mathfrak{g}$ est un isomorphisme de $\mathfrak{g}$ sur l'algèbre de Lie de $G$. On identifie $\mathfrak{g}$ à son image par la représentation adjointe. En particulier, $\mathfrak{g}(\xi)$ est l'algèbre de Lie du stabilisateur $G(\xi)$ de $\xi$ dans $G$ et pour toute forme linéaire régulière $v^{\prime} \operatorname{sur} \mathfrak{g}(\xi), \mathfrak{s}\left(v^{\prime}\right)$ est l'algèbre de Lie du plus grand tore contenu dans le stabilisateur $G(\xi)\left(v^{\prime}\right)$ de $v^{\prime}$ dans $G(\xi)$. On dira que $\mathfrak{s}\left(v^{\prime}\right)$ est le plus grand tore de $\mathfrak{g}(\xi)\left(v^{\prime}\right)$.

LEMME 5.8. - Soit $\mathfrak{s}$ le plus grand tore du stabilisateur d'une forme linéaire fortement régulière sur $\mathfrak{g}(\xi)$. On note $\mathfrak{a}$ le centralisateur de $\mathfrak{s}$ dans $\mathfrak{g}$.

(i) L'élément $\xi$ appartient à l'algèbre dérivée de a.

(ii) La sous-algèbre $\mathfrak{g}(\xi)$ est somme directe de ses intersections respectives $\mathfrak{g}(\xi)_{0}$ et $\mathfrak{g}(\xi)_{+}$avec $\mathfrak{a}$ et $[\mathfrak{s}, \mathfrak{g}]$.

(iii) Les algèbres $\mathfrak{g}(\xi)_{0}$ et $\mathfrak{g}(\xi)$ ont même indice.

(iv) Le centre de a est égal à $\mathfrak{s}$.

(v) On suppose que $[\mathfrak{a}, \mathfrak{a}]$ contient $\rho$ et $\eta$. Soit $\mathfrak{g}(\eta)_{0}^{\prime}$ le centralisateur de $\eta$ dans $[\mathfrak{a}, \mathfrak{a}]$. Alors $\mathfrak{s}$ est le centralisateur dans $\mathfrak{g}(\xi)_{0}$ de $\xi+x$ pour tout $x$ dans un ouvert non vide de $\mathfrak{g}(\eta)_{0}^{\prime}$.

Démonstration. - (i) Puisque $\xi$ appartient au centre de $\mathfrak{g}(\xi), \xi$ centralise $\mathfrak{s}$ car $\mathfrak{s}$ est contenu dans $\mathfrak{g}(\xi)$; donc $\mathfrak{a}$ contient $\xi$. Puisque $\mathfrak{a}$ est une sous-algèbre réductive dans $\mathfrak{g}, \mathfrak{a}$ est somme directe de son algèbre dérivée $[\mathfrak{a}, \mathfrak{a}]$ et du centre $\mathfrak{z} \mathfrak{a}$ de $\mathfrak{a}$. En outre, tout élément de $\mathfrak{z} \mathfrak{a}$ est semi-simple; donc $[\mathfrak{a}, \mathfrak{a}]$ contient $\xi$ car $\xi$ est nilpotent. 
(ii) Le sous-espace $[\mathfrak{s}, \mathfrak{g}]$ de $\mathfrak{g}$ est somme directe des sous-espaces propres de l'action adjointe de $\mathfrak{s}$ dans $\mathfrak{g}$ qui ne sont pas contenus dans $\mathfrak{a}$. Puisque $\mathfrak{g}(\xi)$ contient $\mathfrak{s}, \mathfrak{g}(\xi)$ est stable par l'action adjointe de $\mathfrak{s} ;$ donc $\mathfrak{g}(\xi)$ est somme directe de $\mathfrak{g}(\xi)_{0}$ et de $\mathfrak{g}(\xi)_{+}$.

(iii) Soit $v^{\prime}$ une forme linéaire fortement régulière sur $\mathfrak{g}(\xi)$ pour laquelle $\mathfrak{s}$ est le plus grand tore de $\mathfrak{g}(\xi)\left(v^{\prime}\right)$. Puisque $\mathfrak{g}(\xi)\left(v^{\prime}\right)$ est une sous-algèbre commutative, $\mathfrak{g}(\xi)\left(v^{\prime}\right)$ est contenu dans $\mathfrak{g}(\xi)_{0}$. En outre, $v^{\prime}$ est nul sur $\mathfrak{g}(\xi)_{+} \operatorname{car} \mathfrak{g}(\xi)_{+}$ est égal à $[\mathfrak{s}, \mathfrak{g}(\xi)]$. Si $\nu$ est un élément de $\mathfrak{g}(\xi)_{0}$ qui stabilise la restriction de $v^{\prime}$ à $\mathfrak{g}(\xi)_{0}$, il appartient à $\mathfrak{g}(\xi)\left(v^{\prime}\right)$ car $\mathfrak{g}(\xi)_{+}$contient $\left[\mathfrak{g}(\xi)_{0}, \mathfrak{g}(\xi)_{+}\right]$; donc $\mathfrak{g}(\xi)\left(v^{\prime}\right)$ est le stabilisateur dans $\mathfrak{g}(\xi)_{0}$ de la restriction de $v^{\prime}$ à $\mathfrak{g}(\xi)_{0}$. Par suite, l'indice de $\mathfrak{g}(\xi)_{0}$ est inférieur à l'indice de $\mathfrak{g}(\xi)$. Soient $\nu_{1}, \ldots, \nu_{m}$ une base de $\mathfrak{g}(\xi)_{+}$et $p$ l'élément de $\mathrm{S}(\mathfrak{g}(\xi))$

$$
\operatorname{det}\left(\begin{array}{ccc}
{\left[\nu_{1}, \nu_{1}\right]} & \cdots & {\left[\nu_{1}, \nu_{m}\right]} \\
\vdots & \ddots & \vdots \\
{\left[\nu_{m}, \nu_{1}\right]} & \cdots & {\left[\nu_{m}, \nu_{m}\right]}
\end{array}\right)
$$

Si $v^{\prime}$ est un zéro de $p$, il existe un élément non nul $\nu$ de $\mathfrak{g}(\xi)_{+}$qui satisfait les égalités

$$
\left\langle v^{\prime},\left[\nu, \nu_{1}\right]\right\rangle=\cdots=\left\langle v^{\prime},\left[\nu, \nu_{m}\right]\right\rangle=0 .
$$

Cet élément $\nu$ appartient à $\mathfrak{g}(\xi)\left(v^{\prime}\right)$ car $\mathfrak{g}(\xi)_{+}$contient $\left[\nu, \mathfrak{g}(\xi)_{0}\right]$; donc $p$ n'est pas nul en $v^{\prime}$. Identifiant le dual de $\mathfrak{g}(\xi)_{0}$ à l'orthogonal de $\mathfrak{g}(\xi)$ + dans $\mathfrak{g}(\xi)^{*}$, il en résulte que $\mathfrak{g}(\xi)_{0}^{*}$ n'est pas contenu dans la variété des zéros de $p$; il existe donc une forme linéaire régulière $w^{\prime} \operatorname{sur} \mathfrak{g}(\xi)_{0}$ qui n'est pas un zéro de $p$. Alors $\mathfrak{s}$ stabilise $w^{\prime}$ et $\mathfrak{g}(\xi)\left(w^{\prime}\right)$ est contenu dans $\mathfrak{g}(\xi)_{0}$; donc $\mathfrak{g}(\xi)\left(w^{\prime}\right)$ est le stabilisateur de la forme linéaire $w^{\prime}$ sur $\mathfrak{g}(\xi)_{0}$ car $w^{\prime}$ est nul sur $\left[\mathfrak{g}(\xi)_{0}, \mathfrak{g}(\xi)_{+}\right]$. Il en résulte que l'indice de $\mathfrak{g}(\xi)$ est inférieur à l'indice de $\mathfrak{g}(\xi)_{0}$, d'où l'assertion.

(iv) Par définition, $\mathfrak{s}$ est contenu dans le centre $\mathfrak{z} \mathfrak{a}$ de $\mathfrak{a}$. Si $\nu$ est un élément de $\mathfrak{z} \mathfrak{a}, \nu$ est un élément semi-simple du centre de $\mathfrak{g}(\xi)_{0} ;$ donc $\nu$ appartient au stabilisateur de la restriction de $v^{\prime}$ à $\mathfrak{g}(\xi)_{0}$. Puisque $\mathfrak{s}$ est le plus grand tore de $\mathfrak{g}(\xi)\left(v^{\prime}\right), \mathfrak{s}$ contient $\nu$ car d'après (iii), $\mathfrak{g}(\xi)\left(v^{\prime}\right)$ est le stabilisateur de la restriction de $v^{\prime}$ à $\mathfrak{g}(\xi)_{0}$, d'où l'assertion.

(v) D'après l'assertion (i), $[\mathfrak{a}, \mathfrak{a}]$ contient $\xi$; donc on peut trouver un $\mathfrak{s l}_{2}$ triplet $\xi, \rho, \eta$ dans $[\mathfrak{a}, \mathfrak{a}]$. Pour tout $x$ dans $\mathfrak{g}(\eta)_{0}^{\prime}, \mathfrak{s}$ centralise $\xi+x$. En outre, d'après l'assertion (iv), le centralisateur de $\xi+x$ dans $\mathfrak{g}(\xi)_{0}$ est la somme de $\mathfrak{s}$ et du centralisateur de $\xi+x$ dans l'intersection $\mathfrak{g}(\xi)_{0}^{\prime}$ de $\mathfrak{g}(\xi)_{0}$ et de $[\mathfrak{a}, \mathfrak{a}]$. Pour tout $x$ dans $\mathfrak{g}(\eta)_{0}^{\prime}$, le centralisateur de $x$ dans $\mathfrak{g}(\xi)_{0}^{\prime}$ est égal au centralisateur de $\xi+x$ dans $\mathfrak{g}(\xi)_{0}^{\prime}$. D'après le lemme $5.6, \mathfrak{g}(\eta)_{0}^{\prime}$ est isomorphe au dual de $\mathfrak{g}(\xi)_{0}^{\prime}$ et pour tout $x$ dans $\mathfrak{g}(\eta)_{0}^{\prime}$, le centralisateur de $x$ dans $\mathfrak{g}(\xi)_{0}^{\prime}$ est contenu dans le stabilisateur dans $\mathfrak{g}(\xi)_{0}^{\prime}$, pour l'action coadjointe de $\mathfrak{g}(\xi)_{0}^{\prime}$, de l'image de $x$ par cet isomorphisme. Puisque l'intersection de $\mathfrak{s}$ et de $\mathfrak{g}(\xi)_{0}^{\prime}$ est nulle, pour tout $x$ dans un ouvert non vide de $\mathfrak{g}(\eta)_{0}^{\prime}$, le centralisateur de $\xi+x$ dans $\mathfrak{g}(\xi)_{0}^{\prime}$ ne contient pas d'élément semi-simple non nul; or d'après le corollaire 5.7 ,

TOME $132-2004-\mathrm{N}^{\mathrm{O}} 4$ 
pour tout $x$ dans un ouvert non vide de $\mathfrak{g}(\eta)_{0}^{\prime}, \xi+x$ est un élément semisimple régulier de $[\mathfrak{a}, \mathfrak{a}]$; donc pour tout $x$ dans un ouvert non vide de $\mathfrak{g}(\eta)_{0}^{\prime}$, le centralisateur de $\xi+x$ dans $\mathfrak{g}(\xi)_{0}^{\prime}$ est nul, d'où l'assertion.

Démonstration. - On revient à la démonstration du théorème 5.5. On traite ici le cas où la sous-algèbre $\mathfrak{s}$ est non nulle. D'après l'assertion (iii) du lemme 5.8, l'indice de $\mathfrak{g}(\xi)$ est égal à l'indice de l'intersection $\mathfrak{g}(\xi)_{0}$ de $\mathfrak{a}$ et de $\mathfrak{g}(\xi)$; or $\mathfrak{g}(\xi)_{0}$ est le centralisateur de $\xi$ dans $\mathfrak{a}$ et $[\mathfrak{a}, \mathfrak{a}]$ est de dimension strictement inférieure à la dimension de $\mathfrak{g}$ car $\mathfrak{s}$ est non nul; donc d'après l'hypothèse de récurrence pour $[\mathfrak{a}, \mathfrak{a}]$, le rang de $\mathfrak{g}$ est égal à l'indice de $\mathfrak{g}(\xi)$ car $\mathfrak{a}$ et $\mathfrak{g}$ ont même rang.

Dans la suite de cette section, on supposera que $\xi$ est un élément nilpotent non régulier qui satisfait la condition suivante : le stabilisateur dans $\mathfrak{g}(\xi)$ d'une forme linéaire régulière sur $\mathfrak{g}(\xi)$ est une sous-algèbre de $\mathfrak{g}$ dont les éléments sont nilpotents.

5.5. Soient $W$ un ouvert affine de $\xi+\mathfrak{g}(\eta)$ qui contient $\xi$ et qui satisfait la condition du corollaire 5.7, $\pi$ la restriction de $\pi_{\xi}$ à $G \times W, U$ l'image de $\pi, \mathfrak{C}$ la sous-variété des points $(x, \nu)$ de $U \times \mathfrak{g}$ pour lesquels $\mathfrak{g}(x)$ contient $\nu$ et $\mathfrak{D}$ l'image réciproque de $\mathfrak{C}$ par l'application $\pi \times \mathrm{id}_{\mathfrak{g}}$ où $\mathrm{id}_{\mathfrak{g}}$ désigne le morphisme identité de la variété $\mathfrak{g}$. On note $\mathcal{I}$ l'idéal de définition de $\mathfrak{C}$ dans $\mathcal{O}_{U \times \mathfrak{g}}$ et $\mathcal{J}$ l'idéal de définition de $\mathfrak{D}$ dans $\mathcal{O}_{G \times W \times \mathfrak{g}}$.

LEMme 5.9. - Soit $M$ le module des applications régulières $\varphi$ de $G \times W$ dans $\mathfrak{g}$ qui satisfont la condition suivante : pour tout $(g, x)$ dans $G \times W,[g(x), \mathfrak{g}]$ contient $\varphi(g, x)$. Soit $R$ le module des applications régulières $\varphi$ de $\mathfrak{g}$ dans $\mathfrak{g}$ qui satisfont la condition suivante : pour tout $x$ dans $\mathfrak{g},[x, \mathfrak{g}]$ contient $\varphi(x)$. On note $\mathcal{M}$ la localisation de $M$ sur $G \times W$ et $\mathcal{R}$ la restriction à $U$ de la localisation de $R$ sur $\mathfrak{g}$.

(i) Les idéaux $\mathcal{J}$ et $\left(\pi \times \mathrm{id}_{\mathfrak{g}}\right)^{*}(\mathcal{I})$ de $\mathcal{O}_{G \times W \times \mathfrak{g}}$ sont égaux.

(ii) Les sous-modules $\pi^{*}(\mathcal{R})$ et $\mathcal{M}$ de $\mathcal{O}_{G \times W} \otimes_{\mathbb{C}} \mathfrak{g}$ sont égaux.

Démonstration. - (i) Puisque $\pi$ est un morphisme plat, $\pi \times \mathrm{id}_{\mathfrak{g}}$ est un morphisme plat; donc de la suite exacte de $\mathcal{O}_{U \times \mathfrak{g}}$-modules

$$
0 \rightarrow \mathcal{I} \longrightarrow \mathcal{O}_{U \times \mathfrak{g}} \longrightarrow \mathcal{O}_{\mathfrak{C}} \rightarrow 0,
$$

on tire la suite exacte de $\mathcal{O}_{G \times W \times \mathfrak{g} \text {-modules }}$

$$
0 \rightarrow\left(\pi \times \operatorname{id}_{\mathfrak{g}}\right)^{*}(\mathcal{I}) \longrightarrow \mathcal{O}_{G \times W \times \mathfrak{g}} \longrightarrow\left(\pi \times \mathrm{id}_{\mathfrak{g}}\right)^{*}\left(\mathcal{O}_{\mathfrak{C}}\right) \rightarrow 0 .
$$

Puisque la fonction constante 1 sur $\mathfrak{D}$ est section globale de $\left(\pi \times \operatorname{id}_{\mathfrak{g}}\right)^{*}\left(\mathcal{O}_{\mathfrak{C}}\right)$, $\mathcal{J}$ est contenu dans le noyau du morphisme

$$
\mathcal{O}_{G \times W \times \mathfrak{g}} \longrightarrow\left(\pi \times \mathrm{id}_{\mathfrak{g}}\right)^{*}\left(\mathcal{O}_{\mathfrak{C}}\right)
$$

donc $\mathcal{J}$ est égal à $\left(\pi \times \operatorname{id}_{\mathfrak{g}}\right)^{*}(\mathcal{I})$ car celui-ci est contenu dans $\mathcal{J}$. 
(ii) Soient $x$ un point de $U, y$ un point de la fibre de $\pi$ en $x$ et $v_{1}, \ldots, v_{n}$ une base de $\mathfrak{g}$. On note $\mathcal{R}_{x}$ la fibre de $\mathcal{R}$ en $x, \mathcal{M}_{y}$ la fibre de $\mathcal{M}$ en $y, \mathcal{I}_{(x, 0)}$ la fibre de $\mathcal{I}$ en $(x, 0)$ et $\mathcal{J}_{(y, 0)}$ la fibre de $\mathcal{J}$ en $(y, 0)$. Identifiant $\mathfrak{g}$ à $\mathfrak{g}^{*}$ au moyen de la forme de Killing de $\mathfrak{g}, \mathcal{O}_{U, x} \otimes_{\mathbb{C}} \mathfrak{g}$ est égal au sous-espace des éléments homogènes de degré 1 en $v_{1}, \ldots, v_{n}$ de $\mathcal{O}_{U \times \mathfrak{g},(x, 0)}$.

Puisque l'idéal de $\mathrm{S}(\mathfrak{g})$ engendré par $[x, \mathfrak{g}]$ est l'idéal de définition dans $\mathrm{S}(\mathfrak{g})$ de l'ensemble des points de $\mathfrak{C}$ au-dessus du point $x$ de $U, \mathcal{R}_{x}$ est égal au sousespace des éléments de $\mathcal{I}_{(x, 0)}$ qui sont homogènes de degré 1 en $v_{1}, \ldots, v_{n}$. En outre, $\mathcal{I}_{(x, 0)}$ est contenu dans l'idéal de $\mathcal{O}_{U \times \mathfrak{g},(x, 0)}$ engendré par $v_{1}, \ldots, v_{n}$. De même, $\mathcal{M}_{y}$ est égal au sous-espace des éléments de $\mathcal{J}_{(y, 0)}$ qui sont homogènes de degré 1 en $v_{1}, \ldots, v_{n}$ et $\mathcal{J}_{(y, 0)}$ est contenu dans l'idéal de $\mathcal{O}_{G \times W \times \mathfrak{g},(x, 0)}$ engendré par $v_{1}, \ldots, v_{n}$. En outre, le sous-espace des éléments homogènes de degré 1 en $v_{1}, \ldots, v_{n}$ de $\left(\pi \times \mathrm{id}_{\mathfrak{g}}\right)^{*}(\mathcal{I})_{(y, 0)}$ est le sous- $\mathcal{O}_{G \times W, y^{-m o d u l e}}$ engendré par les éléments $\varphi \circ \pi$ où $\varphi$ est dans $\mathcal{R}_{x} ;$ donc $\mathcal{M}_{y}$ est égal à $\pi^{*}(\mathcal{R})_{y}$ d'après l'assertion (i). $\mathrm{Vu}$ l'arbitraire de $x$ et de $y, \mathcal{M}$ et $\pi^{*}(\mathcal{R})$ sont égaux.

Corollaire 5.10. - Soit $\theta_{0}$ l'endomorphisme $d u \mathbb{C}[W]$-module $\mathbb{C}[W] \otimes_{\mathbb{C}} \mathfrak{g}$,

$$
\varphi \longmapsto(x \mapsto[x, \varphi(x)]) .
$$

Alors l'image de $\theta_{0}$ est égale au module des applications régulières $\varphi$ de $W$ dans $\mathfrak{g}$ qui satisfont la condition suivante: $[x, \mathfrak{g}]$ contient $\varphi(x)$, pour tout $x$ dans $W$.

Démonstration. - On note $N_{0}$ l'image de $\theta_{0}$ et $M_{0}$ le module des applications régulières $\varphi$ de $W$ dans $\mathfrak{g}$ qui satisfont la condition suivante : pour tout $x$ dans $W,[x, \mathfrak{g}]$ contient $\varphi(x)$. Soient $\theta$ l'endomorphisme du $\mathbb{C}[G \times W]$-module $\mathbb{C}[G \times W] \otimes_{\mathbb{C}} \mathfrak{g}$,

$$
\varphi \longmapsto((g, x) \mapsto[g(x), \varphi(g, x)]),
$$

et $N$ son image. Le module $M$ est l'image de $\mathbb{C}[G] \otimes_{\mathbb{C}} M_{0}$ par l'automorphisme $\alpha$ du $\mathbb{C}[G \times W]$-module $\mathbb{C}[G \times W] \otimes \mathbb{C} \mathfrak{g}$,

$$
\varphi \longmapsto((g, x) \longmapsto g \circ \varphi(g, x)) .
$$

En outre, $N$ est l'image de $\mathbb{C}[G] \otimes_{\mathbb{C}} N_{0}$ par $\alpha$; donc il suffit de montrer l'égalité de $M$ et de $N$ car $M_{0}$ et $N_{0}$ s'identifient canoniquement respectivement aux sous-espaces des éléments de $\mathbb{C}[G] \otimes_{\mathbb{C}} M_{0}$ et de $\mathbb{C}[G] \otimes_{\mathbb{C}} N_{0}$ dont la valeur en $(g, x)$ ne dépend pas de $g$. Puisque $G \times W$ est une variété affine, il suffit de montrer que $\mathcal{M}$ est la localisation de $N$ sur $G \times W$.

D'après [2], $R$ est l'image de l'endomorphisme $\tilde{\theta}$ du $\mathrm{S}(\mathfrak{g})$-module $\mathrm{S}(\mathfrak{g}) \otimes_{\mathbb{C}} \mathfrak{g}$,

$$
\psi \longmapsto(x \mapsto[x, \psi(x)]) .
$$

Soient $K$ le noyau de $\tilde{\theta}$ et $\mathcal{K}$ la restriction à $U$ de la localisation sur $\mathfrak{g}$ de $K$. Pour tout $\varphi$ dans $S(\mathfrak{g}) \otimes_{\mathbb{C}} \mathfrak{g}$, l'image de $\varphi \circ \pi$ par $\theta$ est égale à $\tilde{\theta}\left(\varphi^{\prime}\right) \circ \pi$ où $\varphi^{\prime}$

TOME $132-2004-\mathrm{N}^{\mathrm{O}} 4$ 
désigne la restriction de $\varphi$ à $U$. De la suite exacte courte de $\mathcal{O}_{U}$-modules

$$
0 \rightarrow \mathcal{K} \longrightarrow \mathcal{O}_{U} \otimes_{\mathbb{C}} \mathfrak{g} \stackrel{\tilde{\theta}}{\longrightarrow} \mathcal{R} \rightarrow 0,
$$

on déduit alors la suite exacte courte de $\mathcal{O}_{G \times W}$-modules

$$
0 \rightarrow \pi^{*}(\mathcal{K}) \longrightarrow \mathcal{O}_{G \times W} \otimes_{\mathbb{C}} \mathfrak{g} \stackrel{\theta}{\longrightarrow} \pi^{*}(\mathcal{R}) \rightarrow 0,
$$

car $\mathcal{O}_{G \times W}$ est un $\mathcal{O}_{U}$-module plat; donc $\pi^{*}(\mathcal{R})$ est la localisation sur $G \times W$ de $N$. Il résulte alors de l'assertion (ii) du lemme 5.9 que $\mathcal{M}$ est la localisation de $N$ sur $G \times W$.

5.6. On note $X$ l'éclatement en $\xi$ de $W, \sigma$ le morphisme d'éclatement et $\mu$ l'application régulière de $X$ dans l'espace des endomorphismes linéaires de $\mathfrak{g}$ qui au point $x$ associe l'endomorphisme ad $\sigma(x)$. On utilise alors les notations $x \cdot E$, $E(x), \mathrm{i}_{\mu}$ en prenant pour espaces $E$ et $F$ l'espace $\mathfrak{g}$. L'ouvert $X_{\mathrm{r}}$ est l'ensemble des points $x$ de $X$ pour lesquels $E(x)$ est de dimension $\mathrm{i}_{\mu}$ et $X_{*}$ est le plus grand ouvert de $X$ auquel l'application $x \mapsto E(x)$ de $X_{\mathrm{r}}$ dans $\operatorname{Gr}_{\mathrm{i}_{\mu}}(E)$ a un prolongement régulier noté $\alpha_{\mu}$. D'après le corollaire 5.7, l'image de $\sigma$ rencontre l'ensemble des éléments réguliers de $\mathfrak{g} ;$ donc $\mathrm{i}_{\mu}$ est égal au rang de $\mathfrak{g}$ et $\sigma(x)$ est un élément régulier de $\mathfrak{g}$ pour tout $x$ dans $X_{\mathrm{r}}$. En particulier, $\sigma^{-1}(\{\xi\})$ est une hypersurface de $X$, contenue dans $X \backslash X_{\mathrm{r}}$ car $\xi$ n'est pas un élément régulier de $\mathfrak{g}$. D'après l'assertion (i) du lemme 2.3, il existe un ouvert affine $Y$ de $X$ qui rencontre $\sigma^{-1}(\{\xi\})$ et qui satisfait les conditions suivantes :

1) $Y$ est contenu dans $X_{*}$,

2) $Y \backslash X_{\mathrm{r}}$ est une hypersurface lisse, irréductible, contenue dans $\sigma^{-1}(\{\xi\})$ et dont l'idéal de définition dans $\mathbb{C}[Y]$ est engendré par un élément $q$,

$3)$ il existe un sous-espace $\mathfrak{m}$ de $\mathfrak{g}$ qui est un supplémentaire de $\alpha_{\mu}(x)$ dans $\mathfrak{g}$ pour tout $x$ dans $Y$ et qui contient un supplémentaire $\mathfrak{p}$ de $\mathfrak{g}(\xi)$ dans $\mathfrak{g}$.

On désigne par $\mathfrak{m}(\xi)$ l'intersection de $\mathfrak{g}(\xi)$ et de $\mathfrak{m}$, par $\theta$ l'endomorphisme de $\mathbb{C}[Y] \otimes_{\mathbb{C}} \mathfrak{g}$ qui à l'élément $\psi$ associe l'application $x \mapsto \mu(x)(\psi(x))$ et par $\theta_{x}$ l'extension de $\theta$ à $\mathcal{O}_{Y, x} \otimes_{\mathbb{C}} \mathfrak{g}$ pour tout point $x$ de $Y$.

LEMME 5.11. - Soient $Y$ un ouvert affine de $X$ qui satisfait les conditions 1), 2), 3) ci-dessus et $v_{1}, \ldots, v_{s}$ une base de $\mathfrak{m}(\xi)$.

(i) Pour tout $x$ dans $Y \backslash X_{\mathrm{r}}, \mathfrak{g}(\xi)$ est somme directe de $\alpha_{\mu}(x)$ et de $\mathfrak{m}(\xi)$.

(ii) Pour $i=1, \ldots, s$, il existe une application régulière $\nu_{i}$ de $Y$ dans $\mathfrak{g}$ qui satisfait l'égalité $\mu(x)\left(v_{i}\right)=q(x) \nu_{i}(x)$, pour tout $x$ dans $Y$.

(iii) Pour tout $x$ dans un ouvert non vide de $Y \backslash X_{\mathrm{r}}$, l'ensemble des éléments $\nu_{1}(x), \ldots, \nu_{s}(x)$ est une partie libre de $\mathfrak{g}$.

(iv) Soit $\varphi$ une application régulière de $Y$ dans $\mathfrak{g}$ qui est combinaison linéaire à coefficients dans $\mathbb{C}[Y]$ des applications $\nu_{1}, \ldots, \nu_{s}$ et pour laquelle $\mu(x)(\mathfrak{g}(\xi))$

BULLETIN DE LA SOCIÉtÉ MATHÉMATIQUE DE FRANCE 
contient $\varphi(x)$ pour tout $x$ dans $Y$. Alors il existe une application régulière $\psi$ de $Y$ dans $\mathfrak{g}(\xi)$ qui satisfait l'égalité $\varphi(x)=\mu(x)(\psi(x))$, pour tout $x$ dans $Y$.

(v) Soit $M$ le sous-module des éléments $\varphi$ de $\mathbb{C}[Y] \otimes_{\mathbb{C}} \mathfrak{g}$ pour lesquels $x \cdot E$ contient $\varphi(x)$ pour tout $x$ dans $Y$. Alors $M$ est l'image de $\theta$.

Démonstration. - On fixe une base $e_{1}, \ldots, e_{n}$ de $\mathfrak{g}(\eta)$ et on note $x_{1}, \ldots, x_{n}$ la base duale. Pour $i=1, \ldots, n$, on désigne aussi par $x_{i}$ la forme affine sur $\xi+\mathfrak{g}(\eta)$ dont la valeur au point $\xi+x$ de $\xi+\mathfrak{g}(\eta)$ est la valeur de $x_{i}$ en $x$.

(i) Soit $x$ dans $Y \backslash X_{\mathrm{r}}$. Puisque $\mu(x)$ est égal à ad $\xi, \mathfrak{g}(\xi)$ contient $\alpha_{\mu}(x)$; or d'après la condition 3$), \mathfrak{m}$ est un supplémentaire de $\alpha_{\mu}(x)$ dans $\mathfrak{g} ;$ donc $\mathfrak{g}(\xi)$ est somme directe de $\alpha_{\mu}(x)$ et de $\mathfrak{m}(\xi)$.

(ii) Soit $i=1, \ldots, s$. L'application $x \mapsto \mu(x)\left(v_{i}\right)$ de $Y$ dans $\mathfrak{g}$ est nulle en tout point de $Y \backslash X_{\mathrm{r}}$ car $Y \backslash X_{\mathrm{r}}$ est inclus dans $\sigma^{-1}(\{\xi\})$ et $\mathfrak{g}(\xi)$ contient $v_{i}$. L'assertion résulte alors de ce que $q$ engendre l'idéal de définition de $Y \backslash X_{\mathrm{r}}$ dans $\mathbb{C}[Y]$.

(iii) On suppose l'assertion fausse. Il s'agit d'aboutir à une contradiction. Il existe des fonctions régulières non toutes nulles, $a_{1}, \ldots, a_{s}$ sur $Y \backslash X_{\mathrm{r}}$, qui satisfont l'égalité

$$
a_{1}(x) \nu_{1}(x)+\cdots+a_{s}(x) \nu_{s}(x)=0,
$$

pour tout $x$ dans $Y \backslash X_{\mathrm{r}}$. Soit $x$ un point de $Y \backslash X_{\mathrm{r}}$. Quitte à changer de base $e_{1}, \ldots, e_{n}$, on peut supposer que l'ensemble des fonctions

$$
y_{1}=x_{1}, y_{2}=\frac{x_{2}}{x_{1}}, \ldots, y_{n}=\frac{x_{n}}{x_{1}}
$$

est un système de coordonnées de l'anneau local $\mathcal{O}_{X, x}$ de $X$ en $x$. Puisque $x_{1}$ engendre l'idéal de définition de $\sigma^{-1}(\{\xi\})$ dans $\mathcal{O}_{X, x}$, la fonction $q / x_{1}$ est un élément inversible de $\mathcal{O}_{X, x}$ d'après la condition 2) ci-dessus. L'égalité ci-dessus revient à dire qu'il existe des polynômes $p_{1}, \ldots, p_{s}$ en $n-1$ indéterminées, non tous nuls, qui satisfont l'égalité

$$
\begin{aligned}
p_{1}\left(y_{2}, \ldots, y_{n}\right) & {\left[v_{1}, e_{1}+y_{2} e_{2}+\cdots+y_{n} e_{n}\right] } \\
+ & \cdots+p_{s}\left(y_{2}, \ldots, y_{n}\right)\left[v_{s}, e_{1}+y_{2} e_{2}+\cdots+y_{n} e_{n}\right]=0 .
\end{aligned}
$$

Désignant par $d$ le plus grand des degrés des polynômes $p_{1}, \ldots, p_{s}$ et par $\chi$ la fonction polynomiale sur $\mathfrak{g}$,

$$
x_{1}^{d} p_{1}\left(\frac{x_{2}}{x_{1}}, \ldots, \frac{x_{n}}{x_{1}}\right) v_{1}+\cdots+x_{1}^{d} p_{s}\left(\frac{x_{2}}{x_{1}}, \ldots, \frac{x_{n}}{x_{1}}\right) v_{s},
$$

$\chi(x)$ centralise $x$ pour tout $x$ dans $\mathfrak{g}(\eta)$; or d'après l'assertion $(\mathrm{v})$ du lemme 5.8 , pour tout $x$ dans un ouvert non vide de $\mathfrak{g}(\eta)$, le centralisateur de $x$ dans $\mathfrak{g}(\xi)$ est nul car d'après l'hypothèse du lemme sur $\xi, \mathfrak{s}$ est nul et $\mathfrak{g}(\eta)_{0}^{\prime}$ est égal à $\mathfrak{g}(\eta)$; donc $\chi$ est nul. Ceci est absurde car les polynômes $p_{1}, \ldots, p_{s}$ ne sont pas tous nuls.

(iv) Soit $Y_{\mathrm{r}}$ l'intersection de $Y$ et de $X_{\mathrm{r}}$. Il existe une unique application régulière $\psi$ de $Y_{\mathrm{r}}$ dans $\mathfrak{m}$ dont l'image par $\theta$ est la restriction de $\varphi$ à $Y_{\mathrm{r}}$.

TOME $132-2004-\mathrm{N}^{\mathrm{O}} 4$ 
Par hypothèse, il existe des éléments $a_{1}, \ldots, a_{s}$ de $\mathbb{C}[Y]$ qui satisfont l'égalité $\varphi=a_{1} \nu_{1}+\cdots+a_{s} \nu_{s}$. Comme $\varphi$ est nul sur $Y \backslash X_{\mathrm{r}}, q$ divise $a_{1}, \ldots, a_{s}$ dans $\mathbb{C}[Y]$ d'après l'assertion (iii). Désignant par $b_{1}, \ldots, b_{s}$ les quotients respectifs de $a_{1}, \ldots, a_{s}$ par $q$, on a

$$
\varphi(x)=b_{1}(x) \mu(x)\left(v_{1}\right)+\cdots+b_{s}(x) \mu(x)\left(v_{s}\right),
$$

pour tout $x$ dans $Y$ d'après l'assertion (ii) ; donc $\psi$ est une application régulière en tout point $x$ où $\nu_{1}(x), \ldots, \nu_{s}(x)$ sont linéairement indépendants. D'après l'assertion (iii), l'ensemble de ces points est un grand ouvert de $Y$; donc $\psi$ est une application régulière de $Y$ dans $\mathfrak{g}(\xi)$ car $Y$ est une variété normale, d'où l'assertion.

(v) Soient $N$ l'image de $\theta, \mathcal{M}_{x}$ et $\mathcal{N}_{x}$ les localisés au point $x$ de $Y$ des modules $M$ et $N$. Puisque $Y$ est un ouvert affine, il s'agit de montrer que pour tout $x$ dans $Y, \mathcal{M}_{x}$ est égal à $\mathcal{N}_{x}$. Si $x$ n'appartient pas à $\sigma^{-1}(\{\xi\}), \mathcal{M}_{x}$ est égal à $\mathcal{N}_{x}$ d'après le corollaire 5.10 car la restriction de $\sigma$ à $Y \backslash \sigma^{-1}(\{\xi\})$ est un isomorphisme de $Y \backslash \sigma^{-1}(\{\xi\})$ sur un ouvert de $W$. Il suffit alors de montrer l'égalité de $\mathcal{M}_{x}$ et de $\mathcal{N}_{x}$ pour $x$ dans $\sigma^{-1}(\{\xi\})$.

Soit $\varphi$ dans $\mathcal{M}_{x}$. Identifiant l'anneau local $\mathcal{O}_{W, \xi}$ de $W$ en $\xi$ à un sous-anneau de $\mathcal{O}_{X, x}$ au moyen du comorphisme de $\sigma, \mathcal{O}_{W, \xi} \otimes_{\mathbb{C}} \mathfrak{g}$ contient $q^{d} \varphi$ pour $d$ assez grand; donc d'après le corollaire 5.10 , pour $d$ assez grand, $q^{d} \varphi$ appartient à l'image de $\theta_{x}$. Un raisonnement par récurrence sur l'entier $d$ montre alors qu'il suffit de montrer que $\mathcal{N}_{x}$ contient $\varphi$ s'il contient $q \varphi$. Dans ce qui suit, on suppose que $q \varphi$ appartient à $\mathcal{N}_{x}$. Puisque $\mathfrak{g}$ est somme directe de $\mathfrak{m}$ et de $\alpha_{\mu}(y)$ pour tout $y$ dans $Y, q \varphi$ est l'image par $\theta_{x}$ d'un élément $\psi$ de $\mathcal{O}_{X, x} \otimes_{\mathbb{C}} \mathfrak{m}$. On désigne par $\psi_{1}$ et $\psi_{2}$ les composantes de $\psi$ relatives à la décomposition

$$
\mathcal{O}_{X, x} \otimes_{\mathbb{C}} \mathfrak{m}=\mathcal{O}_{X, x} \otimes_{\mathbb{C}} \mathfrak{p} \oplus \mathcal{O}_{X, x} \otimes_{\mathbb{C}} \mathfrak{m}(\xi) .
$$

Alors l'image de $\psi_{2}$ par $\theta$ est divisible par $q$ dans $\mathcal{O}_{X, x} \otimes_{\mathbb{C}} \mathfrak{g}$; donc l'image de $\psi_{1}$ par $\theta$ est divisible par $q$. Il en résulte que $\psi_{1}$ est le produit de $q$ et d'un élément $\psi_{1}^{\prime}$ de $\mathcal{O}_{X, x} \otimes_{\mathbb{C}} \mathfrak{p}$ car l'intersection de $\mathfrak{p}$ et de $\mathfrak{g}(\xi)$ est nulle. Par suite, $\varphi-\theta\left(\psi_{1}^{\prime}\right)$ est combinaison linéaire à coefficients dans $\mathcal{O}_{X, x}$ des applications $\nu_{1}, \ldots, \nu_{s}$; donc d'après l'assertion (iv), $\mathcal{N}_{x}$ contient $\varphi-\theta\left(\psi_{1}^{\prime}\right)$ et $\varphi$, d'où l'assertion.

Fin de la démonstration du théorème 5.5. - La restriction de $\mu$ à $Y$ possède la propriété $(\mathbf{P})$ de la définition 2.2 d'après l'assertion (v) du lemme 5.11. Puisque $\sigma$ est le morphisme d'éclatement de $W$ centré en $\xi, \sigma$ est un morphisme dominant, les fibres de $\sigma$ sont irréductibles et l'espace tangent à $W$ en un point $x$ de $W$ est la réunion des images des applications linéaires tangentes à $\sigma$ en les points de la fibre de $\sigma$ en $x$; or $\mu$ est la composée de $\sigma$ et de l'isomorphisme linéaire $x \mapsto$ ad $x$; donc le morphisme $\mu$ a les propriétés 1 ) et 2) du théorème 3.3. Il résulte alors de ce théorème que $\mu$ a la propriété $(\mathbf{R})$ en tout point d'un grand ouvert de $Y$. Puisque l'intersection de $Y$ et de $\sigma^{-1}(\{\xi\})$ est une hypersurface de $Y, \mu$ a la propriété $(\mathbf{R})$ en un point de $Y$ au-dessus de $\xi$; donc $\mathfrak{g}$ a la 
propriété $(\mathbf{C})$ en $\xi$ au sens de la définition 5.1. Les modules adjoint et coadjoint de $\mathfrak{g}$ sont isomorphes car $\mathfrak{g}$ est semi-simple; donc d'après la proposition 5.2 , l'indice de $\mathfrak{g}(\xi)$ est égal au rang de $\mathfrak{g}$.

\section{BIBLIOGRAPHIE}

[1] Bernat (P.) et al. - Représentations des groupes de Lie résolubles, Monographies de la Société Mathématique de France, vol. 4, Dunod, 1972.

[2] Dixmier (J.) - Champs de vecteurs adjoints sur les groupes et algèbres de Lie semi-simples, J. reine angew. Math., t. 309 (1979), pp. 178-190.

[3] Panyushev (D.I.) - The index of a Lie algebra, the centraliser of a nilpotent element, and the normaliser of the centraliser, arXiv:math.AG/ 0107031, 2001.

[4] Richardson (R.W.) - Commuting varieties of semisimple Lie algebras and algebraic groups, Compositio Math., t. 38 (1979), pp. 311-322.

[5] Shafarevich (I.R.) - Basic Algebraic Geometry 2, Springer-Verlag, 1994.

[6] Slodowy (P.) - Simple singularities and simple algebraic groups, Lecture Notes in Mathematics, vol. 815, Springer-Verlag, 1980.

[7] Tauvel (P.) - Sur les éléments réguliers dans les algèbres de Lie réductives, Bull. Sci. Math., t. 113 (1989), pp. 51-83. 